\title{
PAPR reduction scheme with efficient embedded signaling in MIMO-OFDM systems
}

\author{
Mouna Sghaier ${ }^{*}$, Fatma Abdelkefi ${ }^{*}$ and Mohamed Siala*
}

\begin{abstract}
Multiple input multiple output (MIMO) orthogonal frequency division multiplexing (OFDM) is a promising transmission scheme for high performance broadband wireless communications. However, this technique suffers from a major drawback which is the high peak to average power ratio (PAPR) of the output signals. In order to overcome this issue, several methods requiring the transmission of explicit side information (SI) bits have been proposed in the literature. In fact, the transmitted bits must be channel-encoded as they are particularly critical to the performance of the considered OFDM system. Consequently, this channel-encoding highly increases the system complexity and decreases the transmission data rate. To overcome these problems, we propose in this paper two robust blind techniques that embed the SI implicitly into the OFDM frame. The first technique, referred to as Blind Space Time Bloc Codes (BSTBC), is inspired from the conventional selected mapping (SLM) approach. This technique banks on an adequate embedded signaling that mainly consists in a specific Space Time Bloc Codes (STBC) patterns and a precoding sequence codebook. In addition, in order to improve the signal detection process and the PAPR gain, we propose a new efficient combined Blind SLM-STBC (BSLM-STBC) method. Both methods propose an optimized scheme during the signal estimation process that is based on the maximum a posteriori (MAP) algorithm. A simulation study shows that our investigated approaches result in a spectacular PAPR reduction and furthermore lead to a perfect signal recovery at the receiver side.
\end{abstract}

Keywords: OFDM; PAPR; Selected mapping (SLM); Alamouti algorithm; Space Time Bloc Codes (STBC); Maximum a posterior (MAP); MIMO; Side information (SI); Max-log approximation; Rotated and unrotated quadrature amplitude modulation (QAM); Channel coding; Embedded signaling

\section{Introduction}

Orthogonal frequency division multiplexing (OFDM) modulation technique is a multicarrier transmission scheme that recently has been widely adopted in various wireless communication standards (WLAN, DVBT...), thanks to its high spectral efficiency and robustness offered, especially for the frequency selective channels $[1,2]$. This technique has also been combined with the multiple input multiple output (MIMO) systems [3] and has recently been adopted for many important wireless standards such as the Third Generation Partnership Project (3 GPP) of long-term evolution advanced (LTE-A) [4]. However, the transmitted signals in OFDM systems may potentially have high peak values in the time

\footnotetext{
*Correspondence: mouna.sghaier@supcom.tn; fatma.abdelkefi@supcom.tn; mohamed.siala@supcom.tn

MEDIATRON Laboratory, Sup'com, Tunis, Tunisia
}

domain since many independent subcarrier components are added via an inverse fast Fourier transform (IFFT) operation. This aspect may considerably increase the peak power at the output of the high-power amplifier (HPA) at the transmitter $[5,6]$.

Since high peak values can occur in any transmitted branch of the MIMO-OFDM system, this issue becomes more serious mainly when using several transmitter antennas. It is for this reason that several research works were interested in the PAPR reduction by applying existing single input single output (SISO) algorithms separately on each transmit antenna such as ordinary selected mapping (SLM) [7] and partial transmit sequence (PTS) techniques [8]. Such techniques need a number of side information (SI) equal to the number of used sequence phase for each antenna. Therefore, the SI increases highly with the number of antenna which weakness this approach. To overcome this problem,

\section{望 Springer}

(c) 2015 Sghaier et al. Open Access This article is distributed under the terms of the Creative Commons Attribution 4.0 International License (http://creativecommons.org/licenses/by/4.0/), which permits unrestricted use, distribution, and reproduction in any medium, provided you give appropriate credit to the original author(s) and the source, provide a link to the Creative Commons license, and indicate if changes were made. 
interesting solutions are proposed in the literature such as cross-antenna rotation and inversion [9], direct SLM [10], direct PTS (dPTS) [11], unitary rotation [12], optimal PAPR reduction [13], and polyphase interleaving and inversion [14]. These techniques consist of selecting the transmitted sequence with the lowest average PAPR over all transmit antennas. However, all these methods decrease the SI but imply an explicit SI transmission to recover the useful data at the receiver side. As such, these approaches cause a resource wasting (in terms of channel bandwidth) that can considerably decrease the data rate. Moreover, a wrong estimation of the SI at the received side could damage the total signal recovery which leads to significant performance deterioration in terms of bit error rate (BER). Thus, several recent research works, known as blind techniques [15-17], have been proposed to avoid the sending of the explicit SI. Some of these studies required the use of a special phase rotations of candidate signals $(0$ or $\pi)$ [15], which degrade the PAPR performance. The other proposed approaches are only relevant for two antennas [16] or are too complex to be implemented [17].

This paper focus on avoiding the transmission of the explicit SI in MIMO-OFDM systems. To achieve this goal, we propose two efficient blind methods. In the first part, we propose a new blind technique, one inspired from classical SLM techniques, referred to as Blind Space Time Bloc Codes (BSTBC). This method requires, at the transmitter side, a special precoder codebook containing different configurations of two Space Time Bloc Codes (STBC) patterns. Among them, the one leading to the minimum PAPR will be kept in the PAPR reduction process. Consequently, this leads to an embedded signaling that guarantees a reliable and perfect signal recovery at the receiver side through a hard or a soft decision process. The second method consists of combining the BSTBC method with BSLM scheme already investigated in [18]. This combined method, referred to as Blind SLM-STBC (BSLM-STBC), exploits two forms of signaling which are the set of rotated and unrotated constellations and a precoders codebook containing different configurations of STBC patterns. In this paper, we show that BSLM-STBC approach does not only lead to significant reduce of the PAPR level but also enhances the recovery process at the receiver side. Furthermore, both methods consider a MAX-Log-MAP estimation technique which takes an optimal advantage from these embedded signaling. Compared to the existing works, our proposed methods have the advantage to consider an embedded signaling process that exploits both the transmitter and the receiver sides to jointly reduce the PAPR and guarantee a perfect signal reconstitution without use of an explicit SI.

The remainder of this paper is organized as follows. First, we define the system model in Section 3. Then, we review the conventional SLM technique used in SISO-OFDM system. In Section 4, we describe our proposed blind techniques mainly BSTBC and BSLM-STBC and detail their improved version in Section 5. Subsequently, we present in Section 6 the simulation results to illustrate the performance of our proposed methods. Finally, Section 7 presents the conclusions of this study and makes some suggestions for future work.

\section{Notations}

Throughout this paper, the boldface lower case and upper cases letters denote vectors and matrices respectively. $\mathbf{I}_{N}$ refers to the identity matrix of dimension $N$. The superscripts.$^{T}$ and.$^{*}$ denote the transpose and the element wise conjugation, respectively. Finally, $\mathbb{E}$ refers to the expectation operator, |.| denotes the absolute value, and $\operatorname{Pr}$ refers to the probability.

\section{MIMO-OFDM system, PAPR metric, and SLM approach}

Recently, OFDM combined with MIMO technology has received a great deal of attention since it represents an interesting candidate for mobile communication systems thanks to its robustness to multipath fading channels, ability to achieve high data rate and very high bandwidth efficiencies [20, 21]. Moreover, in order to attain optimum performance in MIMO-OFDM systems, several kinds of diversity (time, frequency, channel coding) are often used. Furthermore, authors in [19] have shown that the performance of OFDM systems using Space-Time Coding (STC) yielded significant gains $(\sim 5 \mathrm{~dB})$ in terms of BER in a multi path channel with a $30-\mathrm{Hz}$ Doppler spectrum and the absence of any time-interleaving. These codes can be classified into STBC and space-time trellis codes (STTCs) [22].

The STBC utilizes the orthogonality property of the used code to achieve full diversity, yet it cannot achieve full-rate transmission when the number of transmit antennas is greater than two [21]. On the other hand, the STBC can guarantee full diversity by the use of enough trellis coding, but the decoding complexity increases exponentially with the number of transmit antennas $[34,35]$. For these reasons, we consider the STBC code in this work. The first and well-known STBC is the Alamouti code, which is an orthogonal STC and has the advantage to be full rate contrarily to those proposed by Tarokh et al. $[23,24]$.

In the sequel, in order to simplify the description of our proposed methods, we focus on the Alamouti codes. Then, the code of rate $\frac{3}{4}$ will be used for the related simulations. It is important to mention that the study of the blind PAPR reduction scheme described in this paper can be generalized to include both of the two previously cited categories of codes. 


\subsection{System model}

For simplicity of presentation, we consider a multiple input single output (MISO) system with two transmit and one receive antennas using an Alamouti code [25] denoted as $S T B C_{2}$.

As shown in Fig.1, the input data block $\mathbf{X}=$ $\left[X_{0}, \ldots, X_{N-1}\right]^{T}$ is first encoded by one possible spacetime codeword matrix into two vectors $\mathbf{X}^{(1)}$ and $\mathbf{X}^{(2)}$ where

$$
\mathbf{X}^{(i)}=\left[\mathbf{a}_{0}^{(i)}, \ldots, \mathbf{a}_{\frac{N}{2}-1}^{(i)}\right]^{T}, i=1,2 .
$$

We note that the Alamouti code word generated by $\mathrm{C}_{n}^{(S T B C)}$ is given by the following expression:

$$
\begin{aligned}
\mathbf{C}_{n}^{(S T B C)} & =\left(\begin{array}{c}
\mathbf{a}_{n}^{(1)} \\
\mathbf{a}_{n}^{(2)}
\end{array}\right)=\left(\begin{array}{cc}
X_{2 n} & -X_{2 n+1}^{*} \\
X_{2 n+1} & X_{2 n}^{*}
\end{array}\right), \\
n & =0, \ldots, \frac{N}{2}-1
\end{aligned}
$$

which verifies

$$
\mathbf{C}_{n}^{(S T B C)}\left(\mathbf{C}_{n}^{(S T B C)}\right)^{H}=\left(\left|X_{2 n}\right|^{2}+\left|X_{2 n+1}\right|^{2}\right) \mathbf{I}_{2},
$$

where $\mathbf{I}_{2}$ is the $2 \times 2$ identity matrix. After performing an $N$-length IFFT, we obtain the two following sequences $\mathbf{x}^{(i)}=\left[x_{0}^{(i)}, \ldots, x_{N-1}^{(i)}\right]^{T}$, such that

$$
x_{m}^{(i)}=\frac{1}{\sqrt{N}} \sum_{n=0}^{N-1} X_{n}^{(i)} e^{j \frac{2 \pi n m}{N}}, i=1,2 \text { and } m=0, \ldots, N-1 .
$$

At the transmitter side, IFFT processing is usually followed by a cyclic prefix (CP) insertion in order to mitigate the intersymbol interference (ISI). Finally, $\mathbf{x}^{(1)}$ and $\mathbf{x}^{(2)}$ are transmitted from the first and the second antennas, respectively.

Regarding the receiver side, we assume that the channel over the two consecutive data symbols is constant during two time slots $T s$, hence $H_{2 n}^{(i)}=H_{2 n+1}^{(i)}, i=1,2$.
In addition, the received signal at time $t$ and $t+T s$, is given by the following expression:

$$
\begin{aligned}
\left(\begin{array}{c}
\mathbf{r}_{2 n} \\
\mathbf{r}_{2 n+1}
\end{array}\right) & =\left(\begin{array}{cc}
X_{2 n} & -X_{2 n+1}^{*} \\
X_{2 n+1} & X_{2 n}^{*}
\end{array}\right) \cdot\left(\begin{array}{c}
H_{2 n}^{(1)} \\
H_{2 n}^{(2)}
\end{array}\right)+\left(\begin{array}{c}
W_{n}^{(1)} \\
W_{n}^{(2)}
\end{array}\right), \\
n & =0, \ldots, \frac{N}{2}-1,
\end{aligned}
$$

where $W_{n}$ are independent and identically distributed (i.i.d.) complex additive white Gaussian noise (AWGN) with zero mean and variance $\sigma^{2}$. In Eq. (4), $H_{n}^{(i)}$ denotes the multipath channel frequency response of the $n^{\text {th }}$ subchannel between the $i^{\text {th }}$ transmitter and the receiver's antenna. Therefore, the Eq. (4) can be expressed as

$$
r_{n}=\sum_{i=1}^{2} X_{n}^{(i)} H_{n}^{(i)}+W_{n}, n=0, \ldots, N-1 .
$$

We also suppose that the channel responses are perfectly estimated at the receiver. Thus, the estimated transmitted symbol can be obtained as

$$
\begin{aligned}
\tilde{X}_{2 n} & =\frac{\left(H_{2 n}^{(1)}\right)^{*} r_{2 n}+H_{2 n}^{(2)} r_{2 n+1}^{*}}{\left\|H_{2 n}^{(1)}\right\|^{2}+\mid H_{2 n}^{(2)} \|^{2}} \text { and } \\
\tilde{X}_{2 n+1} & =\frac{\left(H_{2 n}^{(2)}\right)^{*} r_{2 n}-H_{2 n}^{(1)} r_{2 n+1}^{*}}{\left|H_{2 n}^{(1)}\left\|^{2}+\mid H_{2 n}^{(2)}\right\|^{2}\right.} .
\end{aligned}
$$

\subsection{PAPR metric}

The conventional expression of the PAPR for the MIMOOFDM system is expressed as

$$
P A P R=\frac{\max _{i=1, N_{t}} \max _{k=0, \ldots, N-1}\left|x_{n}^{(i)}\right|^{2}}{\sum_{i=1}^{N_{t}} \mathbb{E}\left(\left\|\mathbf{x}^{(i)}\right\|^{2}\right)},
$$

where $N_{t}$ denotes the number of the transmitter antennas.

\subsection{SLM technique}

To guarantee that the input signal will be in the linear region of the HPA, many techniques were proposed [5]. Among these solutions, the first and the simplest one

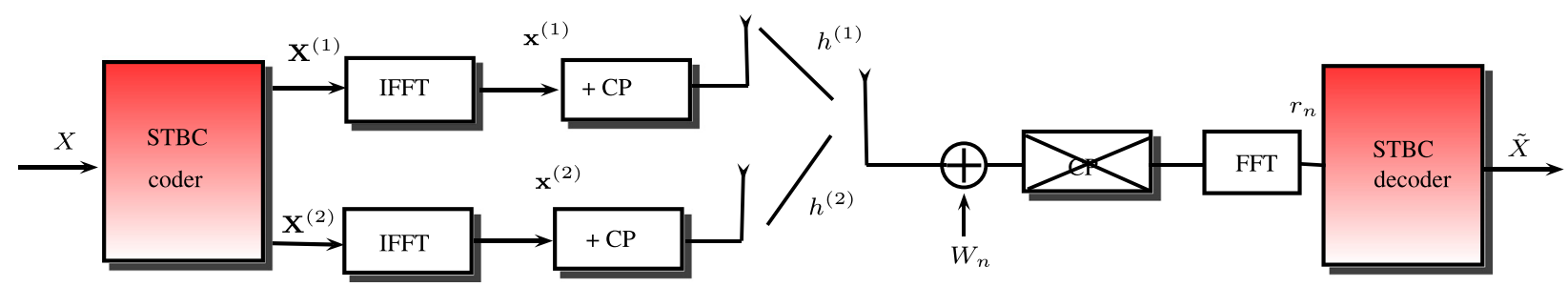

Fig. 1 Proposed system model 
is the clipping of the signal to be amplified [26]. Other methods including coding techniques [27] or based on the relationship between some coding properties and OFDM modulator [28] have been proposed to reduce PAPR. Challenging solutions were proposed such as tone reservation (TR) [29], selected mapping [30], partial transmit sequence [31], etc. Among the cited techniques, we considered in this study the SLM technique due to its ability to reduce the PAPR without causing signal degradation in OFDM system and also thanks to its reduced complexity. Basically, the SLM generates, first, a special $D \times N$ phase matrix denoted as $\mathcal{Z}=\left[e^{-j \phi^{(0)}}, \ldots, e^{-j \phi^{(D-1)}}\right]^{T}$, where, $\boldsymbol{\phi}^{(d)}=\left[\phi_{0}^{(d)}, \phi_{i}^{(d)} \ldots, \phi_{N-1}^{(d)}\right], \phi_{i}^{(d)} \in[0,2 \pi[$, and $i=0, \ldots, N-1$. Then, the input data $\mathbf{r}$ is multiplied by the $D$ independent phase sequences which produce a modified data block denoted by $\mathbf{r}^{(d)}=\mathbf{r} \cdot e^{-j \phi^{(d)}}, d=$ $0, \ldots, D-1$.

Finally, the IFFT of the multiplied sequences are taken to produce the $D$ sequences $\mathbf{x}^{(\mathbf{d})}$ among which the one with the lowest PAPR is selected for the transmission as

$$
\tilde{d}=\underset{d=0, \ldots, D-1}{\arg \min } \frac{\max _{n=0, \ldots, N-1}\left|x_{n}^{(d)}\right|^{2}}{\mathbb{E}\left(\left\|\mathbf{x}^{(\mathbf{d})}\right\|^{2}\right)} .
$$

The main steps of the conventional algorithm are summarized according the following Algorithm 1

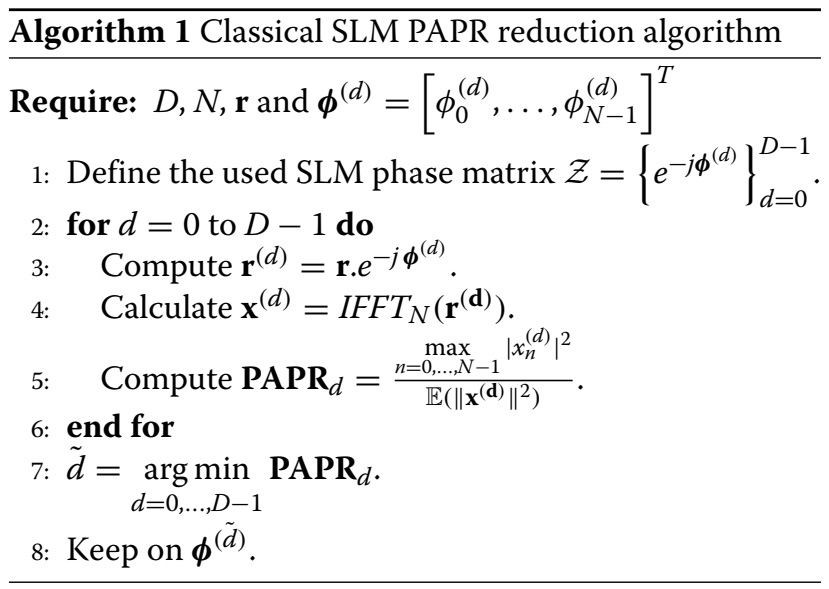

To perform the appropriate inverse operation, the receiver should know which phase sequence is used at the transmitter side. Thus, a portion of the bandwidth must be allocated for the transmission of the SI index. Obviously, $\log _{2}(D)$ bits are required to explicitly represent this SI. Hence, a wrong estimation of it at the received side leads to a damage on the total signal recovery. One alternative may be to protect the SI with some forms of coding techniques, but this will result in further bandwidth loss and resources waste.
For these reasons, we have proposed in our previous work [18] a new Blind SLM (BSLM) for SISO-OFDM systems. The proposed method defines at the transmitter side a specific set $\mathcal{E}$ of the possible candidate phase sequences in order to embed the SI. This set $\mathcal{E}$ induces two subsystems: $\mathcal{E}=\mathcal{E}_{1} \cup \mathcal{E}_{2}$ where $\mathcal{E}_{2}$ is the set of rotated constellation with a specific $\phi$ angle whereas $\mathcal{E}_{1}$ leaves the constellation unchanged. Then, the $D$ candidate phase sequences $\left\{\phi^{(d)}\right\}_{d=0}^{D-1}$, used in conventional SLM, are randomly generated from the set $\mathcal{E}$. This construction allows introducing a new code vector defined as follows:

$$
V_{n}^{(d)}=\left\{\begin{array}{l}
0 \text { if } \phi_{n}^{(d)} \in \mathcal{E}_{1}, \\
1 \text { if } \phi_{n}^{(d)} \in \mathcal{E}_{2}, d=0 \ldots, D-1, n=0, \ldots, N-1 .
\end{array}\right.
$$

This code stands for an embedded signaling which is used to detect the selected sequence phase $\tilde{d}$ at the received side. This is accomplished by comparing the Hamming distance between the received signal and the rotated and unrotated $M$-QAM constellation $(\mathcal{Q}=$ $\left.\left\{q_{0}, \ldots, q_{M-1}\right\}, \mathcal{Q}^{\prime}=\left\{q_{0}^{\prime}, \ldots, q_{M-1}^{\prime}\right\}\right)$ according to the two following hypotheses:

$$
\left\{\begin{array}{l}
\mathcal{H}_{0}: X_{n}^{(d)} \in \mathcal{Q} \Rightarrow \phi_{n}^{(d)} \in \mathcal{E}_{1} \Rightarrow V_{n}^{(d)}=0, \\
\mathcal{H}_{1}: X_{n}^{(d)} \in \mathcal{Q}^{\prime} \Rightarrow \phi_{n}^{(d)} \in \mathcal{E}_{2} \Rightarrow V_{n}^{(d)}=1
\end{array}\right.
$$

At the receiver side, the estimation technique is based on the MAP decoding algorithm that calculates the likelihood ratio $(L R)$ corresponding to each hypothesis $\mathcal{H}_{i}, i=$ 0,1 , with reference to the received symbol $S_{n}$. Then, each component $V_{n}^{(\tilde{d})}$ is estimated through the following metric as

$$
V_{n}^{(\tilde{d})}=\left\{\begin{array}{l}
0 \text { if } L L R_{n}>=0, n=0, \ldots, N-1, \\
1 \text { else }
\end{array}\right.
$$

where $L L R_{n} \approx \min _{l \in \mathcal{Q}}\left|S_{n}-H_{n} q_{l}^{\prime}\right|^{2}-\min _{l \in \mathcal{Q}^{\prime}} \mid S_{n}-$ $\left.H_{n} q_{l}\right|^{2}$, with $H_{n}$ is the frequency response of the multipath channel.

In the next section, we detail our powerful blind method which is an extension of the Blind SLM method conceived to protect the SI. This proposed technique banks on an adequate embedded signaling that mainly consists on a specific STBC patterns and a precoding sequences codebook.

\section{Blind PAPR reduction scheme for MIMO-OFDM systems}

The proposed PAPR reduction technique is based on specific STBC patterns from which we construct precoding sequences forming a codebook that ensures the role of an inherent embedded signaling for the MIMO-OFDM 
system. This technique is blind and banks on the SLM principle. To achieve this objective, first, we start by explaining the construction of this codebook banking on the STBC class. Then, we detail the investigated technique dedicated to reduce the PAPR level. Finally, we illustrate the processing of estimating the SI.

\subsection{Principle of the codebook construction}

In order to embed the SI, we define, at the transmitter side, a binary $K \times \frac{N}{2}$ matrix denoted also a codebook which is the following:

$$
\mathcal{M}=\left[\operatorname{Seq}^{(1)}, \ldots, \operatorname{Seq}^{(K)}\right]^{T}
$$

where $K$ presents the number of precoding sequence. We call a precoding sequence a $1 \times \frac{N}{2}$ binary vector which refers to the way to decode the original data according to the two different Alamouti codes $\left.(\mathbf{S} \mathbf{c h} \text { and } \mathbf{S} \mathbf{c h})_{1}\right)$. To explain more, if $S e q_{n}^{(k)}=0$, then the $\mathbf{S c h}_{0}$ will be used to decode $\left(X_{2 n}, X_{2 n+1}\right)$. Otherwise, we will exploit the other scheme $\mathbf{S c h}_{1}$. This can be summarized as follows:
We note that the choice of the two used schemes is done such as:

- First, we kept only the non-equivalent schemas. For example, for two antennas [25], we can define the non-equivalent schemes as follows:

$$
\begin{aligned}
& \mathbf{A}=\left[\begin{array}{cc}
X_{2 n} & -X_{2 n+1}^{*} \\
X_{2 n+1} & X_{2 n}^{*}
\end{array}\right], \mathbf{B}=\left[\begin{array}{cc}
X_{2 n} & X_{2 n+1}^{*} \\
X_{2 n+1} & -X_{2 n}^{*}
\end{array}\right], \\
& \mathbf{C}=\left[\begin{array}{cc}
X_{2 n} & X_{2 n+1} \\
-X_{2 n+1}^{*} & X_{2 n}^{*}
\end{array}\right], \mathbf{D}=\left[\begin{array}{cc}
X_{2 n} & X_{2 n+1} \\
X_{2 n+1}^{*} & -X_{2 n}^{*}
\end{array}\right] .
\end{aligned}
$$

- Then, for each combination (for two antennas we define six possibilities $A B, A C, A D, B C, B D$, and $C D$ ), we perform the proposed coded scheme (see Eq. (13)) and calculate the PAPR according to the Eq. (7)).

- Finally, we keep the two schemes that lead to the minimum PAPR.

In the case of two antennas, we conclude from Fig. 2 where the curves of the complementary cumulative density function (CCDF) of the PAPR versus its threshold are plotted. We note that a significant gain around $5 d B$

$$
\left\{\begin{array}{l}
\text { if } \operatorname{Seq}_{n}^{(k)}=0 \Rightarrow\left(X_{2 n}, X_{2 n+1}\right), \text { decoded with } \mathbf{S c h}_{0}, \\
\text { if } \operatorname{Seq}_{n}^{(k)}=1 \Rightarrow\left(X_{2 n}, X_{2 n+1}\right), \text { decoded with } \mathbf{S c h}_{1}, n=0, \ldots, \frac{N}{2}-1, k=0, \ldots K-1 .
\end{array}\right.
$$

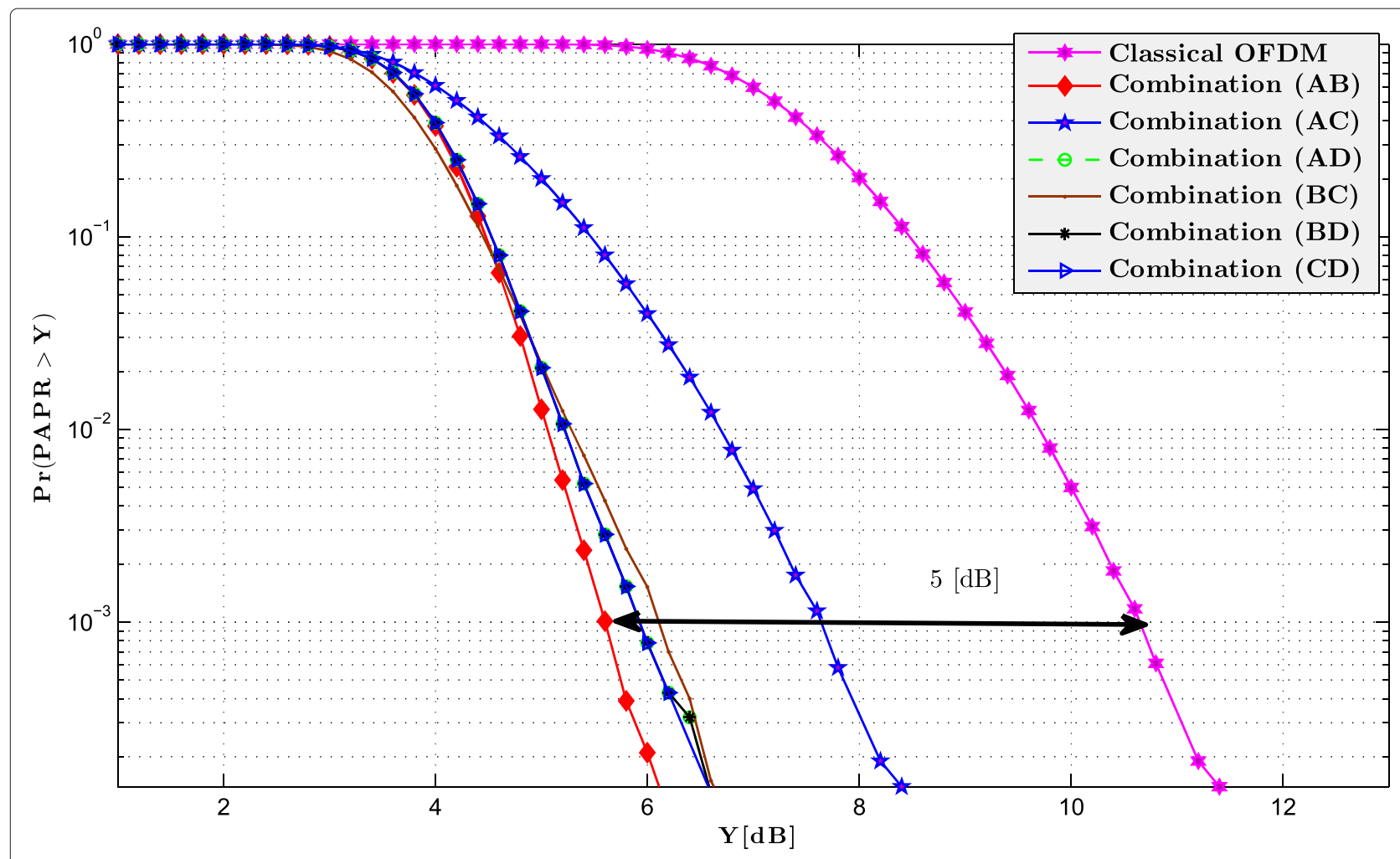

Fig. 2 CCDFs of the PAPR for different two Alamouti codes ( $N=128$ and 4-QAM) 
is observed between the combination $(A B)$ and the classical OFDM scheme for both scenarios, i.e., $N=128$ and 4-QAM constellation.

Consequently, through simulation results, we define the two optimal combined Alamouti codes used in this paper denoted as combination $(A B)$.

$$
\begin{aligned}
& \mathbf{S c h}_{0}=\mathbf{A}:\left(\begin{array}{cc}
X_{2 n} & -X_{2 n+1}^{*} \\
X_{2 n+1} & X_{2 n}^{*}
\end{array}\right) \text { and } \\
& \mathbf{S c h}_{1}=\mathbf{B}:\left(\begin{array}{cc}
X_{2 n} & X_{2 n+1}^{*} \\
X_{2 n+1} & -X_{2 n}^{*}
\end{array}\right) .
\end{aligned}
$$

This can be justified by the fact that these two schemes offer more diversity at the antennas level (see Eq. (14)).

\subsection{PAPR reduction algorithm}

To reduce the PAPR level, we reiterate the same SLM methodology as explained in Section 3.3 where the dictionary of the rotation factor in SLM is equivalent, in our case, to the STBC codebook $\mathcal{M}$ as it is showed in Fig. 3.

To explain the proposed PAPR algorithm, we considered an $S T B C_{2}$ MISO system, where the number of the transmitter antennas $N_{t}=2, N=8, K=3$ and

$$
\mathcal{M}=\left(\begin{array}{llll}
0 & 1 & 1 & 0 \\
1 & 1 & 0 & 1 \\
1 & 0 & 0 & 1
\end{array}\right)=\left(\begin{array}{l}
\mathbf{S e q}^{(1)} \\
\mathbf{S e q}^{(0)} \\
\mathbf{S e q}^{(3)}
\end{array}\right)
$$

Let $\mathbf{X}=\left[X_{0}, X_{1}, X_{2}, X_{3}, X_{4}, X_{N}-1\right]^{T}$ be the transmitted sequence. We recall that each row of $\mathcal{M}$ corresponds to a possible precoding sequence of $\mathbf{X}$ that is encoded into two vectors $\mathbf{X}^{(1)}$ and $\mathbf{X}^{(2)}$. As we consider $\mathbf{S c h}_{0}$ and $\mathbf{S c h}_{1}$ previously defined in Eq. (14), then each precoding sequence Seq ${ }^{(k)}$ leads to one possible coding scheme as follows:

$$
\left(\begin{array}{c}
\mathbf{S e q}^{(1)} \\
\vdots \\
\vdots \\
\mathbf{S e q}^{(K-1)}
\end{array}\right) \Rightarrow\left(\begin{array}{ccccc}
X_{0} & -X_{1}^{*} & \ldots & X_{2 N-2} & -X_{2 N-1}^{*} \\
X_{1} & X_{0}^{*} & \ldots & X_{2 N-1} & X_{2 N-2}^{*} \\
& & \ldots & & \\
& & \ldots & & \\
X_{0} & X_{1}^{*} & \ldots & X_{2 N-2} & -X_{2 N-2}^{*} \\
X_{1} & -X_{0}^{*} & \ldots & X_{2 N-1}^{*} & X_{2 N-1}^{*}
\end{array}\right) \text { where } k=1, \ldots, K .
$$

Then, we kept the precoding sequence Seq ${ }^{\left(k_{0}\right)}$ corresponding to the $k_{0}^{\text {th }}$ row of the precoding matrix leading to the minimum PAPR level according to Eq. (7). Finally, the proposed precoding technique is detailed in Algorithm 2.

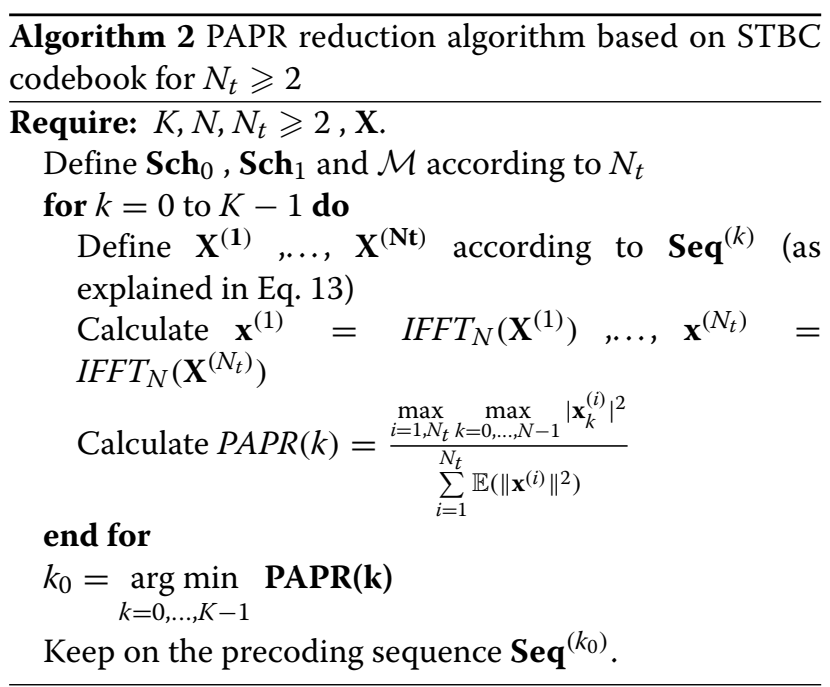

Regarding the receiver side, we propose an efficient decision technique based on the MAP estimator to recover the transmitted sequence $\mathbf{X}$ as it will be explained in the next section.

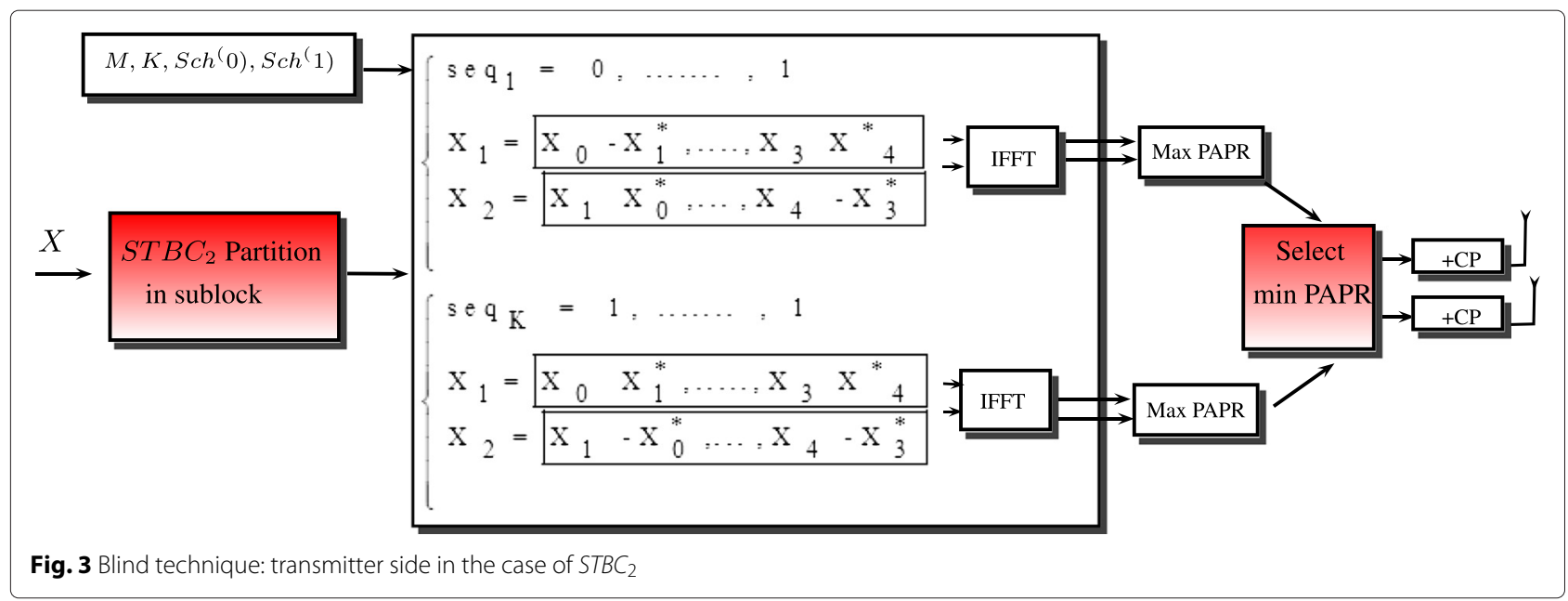




\subsection{Decoding of the side information $k_{0}$}

In this section, we present the block diagram for the recovery of the SI index as shown in Fig. 4.

For the rest of the paper, we assume that both the transmitter and the receiver consider the same codebook $\mathcal{M}$ and that during the recovery process, the channel state information (CSI) is perfectly known. Let $\mathbf{r}=$ $\left[r_{0}, \ldots, r_{N-1}\right]^{T}$ be the received sequence where $r_{n}$ is defined in Eq. (5) and $\mathbf{R}_{n}$ is the couple of the received sequences such that

$$
\mathbf{R}_{n}=\left[r_{2 n}, r_{2 n+1}\right]^{T}, n=0, \ldots, \frac{N}{2}-1 .
$$

It is clear that $r_{n}$ is a function of $\mathbf{X}^{(i)}, i=1,2$, which depends on the used precoder sequence $\mathbf{S e q}^{\left(k_{0}\right)}$ and leads to the minimum value of the PAPR. Subsequently, to efficiently detect the used index, we consider two hypotheses that arise from the methodology used to define the codebook $\mathcal{M}$ in order to encode the couple $\left(X_{2 n}, X_{2 n+1}\right)$ as

$$
\left\{\begin{array}{l}
\mathcal{H}_{0}:\left(X_{2 n}, X_{2 n+1}\right) \text { is encoded according to }\left(\mathbf{S c h}_{0}\right) \\
\mathcal{H}_{1}:\left(X_{2 n}, X_{2 n+1}\right) \text { is encoded according to }\left(\mathbf{S c h}_{1}\right)
\end{array}\right.
$$

In the following paragraph, we explain the hard decision process dedicated to detect the used index $\tilde{k}_{0}$ in an efficient way.

\subsubsection{Hard decision process}

The proposed decision technique uses the MAP decoding algorithm that computes the LR corresponding to each hypothesis $\mathcal{H}_{i}, i=0,1$, based on the two received symbol $\mathbf{R}_{n}$ according to the following expression:

$$
L R_{n}=\frac{\operatorname{Pr}\left(\mathcal{H}_{0} \mid \mathbf{R}_{n}\right)}{\operatorname{Pr}\left(\mathcal{H}_{1} \mid \mathbf{R}_{n}\right)}, n=0, \ldots, \frac{N}{2}-1,
$$

where $\operatorname{Pr}\left(\mathcal{H}_{i} \mid \mathbf{R}_{n}\right)$ is the a posteriori probability (APP) of the hypothesis $\mathcal{H}_{i}, i=0,1$, based on the received symbol
$\mathbf{R}_{n}$. Then, using the Bayes' theorem, the expression of $\operatorname{Pr}\left(\mathcal{H}_{i} \mid \mathbf{R}_{n}\right)$ is as follows:

$$
\operatorname{Pr}\left(\mathcal{H}_{i} \mid \mathbf{R}_{n}\right)=\frac{\operatorname{Pr}\left(\mathbf{R}_{n} \mid \mathcal{H}_{i}\right) \cdot \operatorname{Pr}\left(\mathcal{H}_{i}\right)}{\operatorname{Pr}\left(\mathbf{R}_{n}\right)}, n=0, \ldots, \frac{N}{2}-1 .
$$

Assuming that $\operatorname{Pr}\left(\mathcal{H}_{0}\right)=\operatorname{Pr}\left(\mathcal{H}_{1}\right)=\frac{1}{2}$, Eq. (17) can be expressed as

$$
L R_{n}=\frac{\operatorname{Pr}\left(\mathbf{R}_{n} \mid \mathcal{H}_{0}\right)}{\operatorname{Pr}\left(\mathbf{R}_{n} \mid \mathcal{H}_{1}\right)}, n=0, \ldots, \frac{N}{2}-1
$$

Furthermore, we deduce from the total probability theorem that $\operatorname{Pr}\left(\mathbf{R}_{n} \mid \mathcal{H}_{i}\right)$ has the following expression:

$$
\operatorname{Pr}\left(\mathbf{R}_{n} \mid \mathcal{H}_{i}\right)=\sum_{X_{2 l} \in Q} \sum_{X_{2 l+1} \in Q} \operatorname{Pr}\left(\mathbf{R}_{n} \mid \mathcal{H}_{i}, X_{2 l}, X_{2 l+1}\right), i=0,1
$$

Since $W_{2 n}$ and $W_{2 n+1}$ are assumed to be i.i.d Gaussian variables, thus we can reformulate the last expression as follows

$$
\begin{aligned}
\operatorname{Pr}\left[\mathbf{R}_{n} \mid \mathcal{H}_{0}\right]= & \frac{1}{\pi^{2} \sigma^{4}} \sum_{X_{l} \in Q} \sum_{X_{2 l+1} \in Q} \\
& \exp \left(-\frac{\left|r_{2 l}-H_{2 l}^{(1)} X_{2 l}-H_{2 l}^{(2)} X_{2 l+1}\right|^{2}}{\sigma^{2}}\right) \\
& \times \exp \left(-\frac{\left|r_{2 l+1}+H_{2 l}^{(1)} X_{2 l+1}^{*}-H_{2 l}^{(2)} X_{2 l}^{*}\right|^{2}}{\sigma^{2}}\right)
\end{aligned}
$$

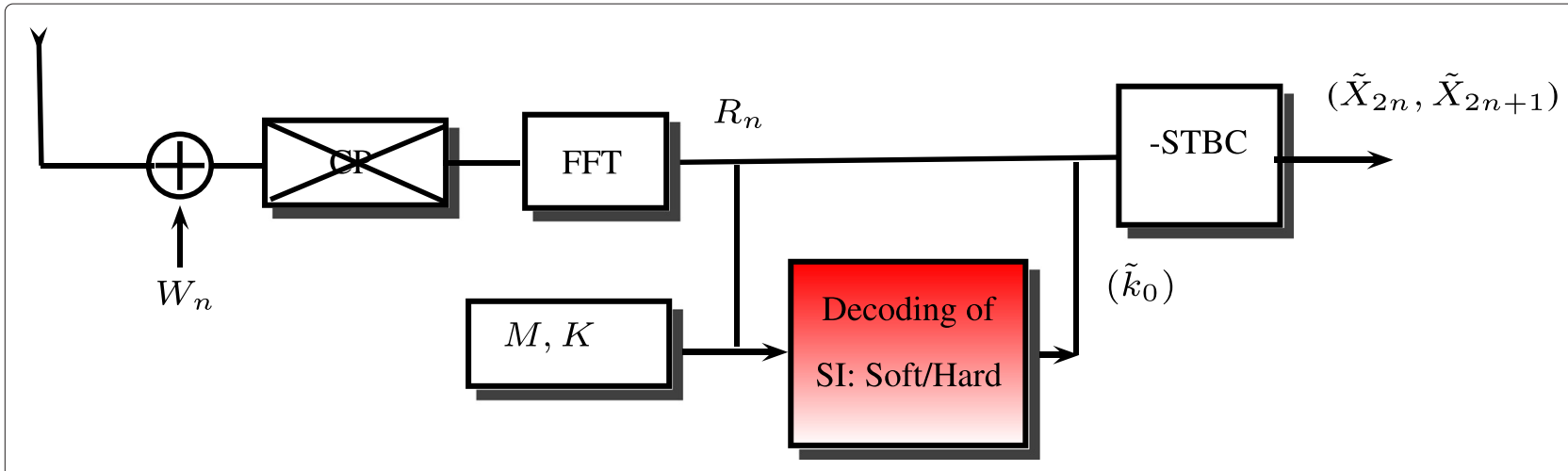

Fig. 4 Block diagram of the receiver for the proposed new Blind STBC technique 
and

$$
\begin{aligned}
\operatorname{Pr}\left[\mathbf{R}_{n} \mid \mathcal{H}_{1}\right]= & \frac{1}{\pi^{2} \sigma^{4}} \sum_{X_{2 l} \in Q} \sum_{X_{2 l+1} \in Q} \\
& \exp \left(-\frac{\left|r_{2 l}-H_{2 l}^{(1)} X_{2 l}-H_{2 l}^{(2)} X_{2 l+1}\right|^{2}}{\sigma^{2}}\right) \\
& \times \exp \left(-\frac{\left|r_{2 k+1}-H_{2 l}^{(1)} X_{2 l+1}^{*}+H_{2 k}^{(2)} X_{2 l}^{*}\right|^{2}}{\sigma^{2}}\right) .
\end{aligned}
$$

It results that the quantity $L R_{n}$ can be obtained as

$$
L R_{n}=\frac{\operatorname{Pr}\left(\mathbf{R}_{n} \mid \mathcal{H}_{0}\right)}{\operatorname{Pr}\left(\mathbf{R}_{n} \mid \mathcal{H}_{1}\right)}=\frac{\sum_{2 l+1} \in Q \sum_{2 l} \in Q}{\sum_{X_{2 l+1} \in Q} \sum_{X_{2 l} \in Q} \exp \left(-f\left(X_{2 l}, X_{2 l+1}\right)\right)},
$$

where $Q=\left\{q_{0}, \ldots, q_{M-1}\right\}$ denotes the set of the considered constellation, including the M-QAM and the QPSK modulations, and the functions $f$ and $g$ are defined as

$$
\begin{gathered}
f\left(X_{2 l}, X_{2 l+1}\right)=\frac{1}{\sigma^{2}}\left(\left|r_{2 l}-H_{2 l}^{(1)} X_{2 l}-H_{2 l}^{(2)} X_{2 l+1}\right|^{2}+\mid r_{2 l+1}\right. \\
\left.+H_{2 l}^{(1)} X_{2 l+1}^{*}-\left.H_{2 l}^{(2)} X_{2 l}^{*}\right|^{2}\right), \\
g\left(X_{2 l}, X_{2 l+1}\right)=\frac{1}{\sigma^{2}}\left(\left|r_{2 l}-H_{2 l}^{(1)} X_{2 l}-H_{2 l}^{(2)} X_{2 l+1}\right|^{2}+\mid r_{2 l+1}\right. \\
\left.-H_{2 l}^{(1)} X_{2 l+1}^{*}+\left.H_{2 l}^{(2)} X_{2 l}^{*}\right|^{2}\right) .
\end{gathered}
$$

Since it is easier to manipulate the $\log$ operator when dealing with the exponential function, the equivalent $L R$ expression becomes equivalent to the $L L R$ that is defined as follows:

$$
L L R_{n}=\ln \left\{\frac{\sum_{X_{2 l+1} \in Q} \sum_{X_{2 l} \in Q} \exp \left(-f\left(X_{2 l}, X_{2 l+1}\right)\right)}{\sum_{X_{2 l+1} \in Q} \sum_{X_{2 l} \in Q} \exp \left(-g\left(X_{2 l}, X_{2 l+1}\right)\right)}\right\} .
$$

In order to avoid the explicit evaluation of the exponential function and to reduce the complexity, we exploit the max-log approximation [32]. Using this approximation, we assume that a single element can be retained in the numerator and the denominator and which has the major contribution in the sum as

$$
\sum_{X_{2 l+1} \in Q} \sum_{X_{2 l} \in Q} \exp \left(-f\left(X_{2 l}, X_{2 l+1}\right)\right) \approx \max _{X_{2 l}, X_{2 l+1} \in Q} e^{-f\left(X_{2 l}, X_{2 l+1}\right)} .
$$

As result, using this approximation, we define the $M L L R_{n}$ metric as

$$
\begin{aligned}
M L L R_{n} & \approx \ln \left\{\frac{\max _{2 l+1}, X_{2 l} \exp \left(-f\left(X_{2 l}, X_{2 l+1}\right)\right)}{\max _{X_{2 l+1}, X_{2 l}} \exp \left(-g\left(X_{2 l}, X_{2 l+1}\right)\right)}\right\} \\
& \approx-\min _{X_{2 l}, X_{2 l+1}} f\left(X_{2 l}, X_{2 l+1}\right)+\min _{X_{2 l}, X_{2 l+1}} g\left(X_{2 l}, X_{2 l+1}\right) .
\end{aligned}
$$

Finally, the proposed decision criteria used to estimate the $\mathbf{S e q}^{\left(k_{0}\right)}$ components is

$\operatorname{Seq}^{\left(k_{0}\right)}=\left\{\begin{array}{l}\operatorname{Seq}_{n}^{\left(k_{0}\right)}=1, \text { if } M L L R_{n} \geq 0 n=0, \ldots, \frac{N}{2}-1 . \\ \operatorname{Seq}_{n}^{\left(k_{0}\right)}=0, \text { else. }\end{array}\right.$

Besides, as the precoder matrix is known at the receiver side, then by using the min-max criteria, the index of the considered precoder sequence $\tilde{k_{0}}$ is deduced according to the following metric:

$$
\tilde{k}_{0}=\underset{0 \leq k \leq K-1}{\arg \min }\left|\mathbf{S e q}^{(k)}-\mathbf{S e q}^{\left(k_{0}\right)}\right| .
$$

Indeed, the hard decision criteria ensures good decision performance, but when it is close to zero (for low signal to noise ratio (SNR) values), making a hard decision based on $\mathcal{H}_{0}$ or $\mathcal{H}_{1}$ could lead to a wrong estimation of the code vector and subsequently introduce a major decision error. To resolve this issue, we investigate, in the following paragraph the soft decision criteria.

\subsubsection{Soft decision process}

To resolve the issue of very low $|L L R|$, we define a new decision in which the estimation of the code vector $\operatorname{Seq}^{(\tilde{k})}$ depends on the whole received sequence $\mathbf{R}_{\mathbf{n}}$ as

$$
\tilde{k}_{0}=\underset{0 \leq k \leq K-1}{\operatorname{argmax}} \operatorname{Pr}\left(\mathbf{R}_{n} \mid \mathbf{S e q}^{(\mathbf{k})}\right) .
$$

To deduce the decision metric, we first begin by defining the closed-form expression of the conditional probability $\operatorname{Pr}\left(\mathbf{R}_{n} \mid \mathbf{S e q}{ }^{(k)}\right)$. Since the precoder sequences $\left\{S e q_{n}^{(k)}\right\}_{n=0}^{N / 2-1}$ are by construction assumed to be i.i.d., therefore, $S e q_{n}^{(k)}$ has no influence on $\mathbf{R}_{n}$. As a results, we obtain

$$
\operatorname{Pr}\left(\mathbf{R}_{n} \mid \mathbf{S e q}^{(\mathbf{k})}\right)=\prod_{n=0}^{N / 2-1} \operatorname{Pr}\left(\mathbf{R}_{n} \mid \operatorname{Seq} q_{n}^{(k)}\right) .
$$

Based on Eq. (30), the index $\tilde{k}_{0}$ can be estimated as 


$$
\begin{aligned}
\tilde{k}_{0} & =\underset{0 \leq k \leq K-1}{\operatorname{argmax}} \operatorname{Pr}\left(\mathbf{R}_{n} \mid \operatorname{Seq}^{(k)}\right) \\
& =\underset{0 \leq k \leq K-1}{\arg \max } \sum_{n=0}^{N / 2-1} \log \left\{\frac{\operatorname{Pr}\left(\mathbf{R}_{n} \mid S e q_{n}^{(k)}\right)}{\operatorname{Pr}\left(\mathbf{R}_{n} \mid \operatorname{Seq}_{n}^{-}{ }^{(k)}\right)}\right\},
\end{aligned}
$$

where $\overline{S e q_{n}^{(k)}}$ is the complementary of $S e q_{n}^{(k)}$.

Finally, we deduce that the estimated index $\tilde{k_{0}}$ can be expressed as follows:

$$
\tilde{k_{0}}=\underset{0 \leq k \leq K-1}{\arg \max } \sum_{n=0}^{\frac{N}{2}-1} \Lambda_{n} \xi_{n}^{(k)}=\underset{0 \leq k \leq K-1}{\arg \max }\left\langle\boldsymbol{\xi}^{(k)}, \boldsymbol{\Lambda}\right\rangle,
$$

where

$$
\xi_{n}^{(k)}=\left\{\begin{array}{ll}
1 & \text { if } S e q_{n}^{(k)}=0, \\
-1 & \text { if } S e q_{n}^{(k)}=1 .
\end{array} \quad \Lambda_{n}=M L L R_{n}, n=0, \ldots, \frac{N}{2}-1\right.
$$

\section{Blind SLM-STBC technique}

In this section, we propose a powerful detection process denoted as "BSLM-STBC" technique. As it is illustrated in Fig. 5, this technique consists on combining both the STBC precoder codebook $\mathcal{M}$ and the set of rotated and unrotated $M$-QAM constellations used in [18]. This embedded signaling enhance the detection process described in the Section 4 and also significantly reduce the PAPR level.

\subsection{Embedding the SI into the OFDM symbol}

The main steps of the improved algorithm performed at the transmitter side are summarized according to the following steps. First, we multiply the original data $\mathbf{X}$ by independent phase sequences to obtain $\mathbf{X}^{\phi}$. Then, each couple $\left(X_{2 n}^{\phi}, X_{2 n+1}^{\phi}\right)$ will be encoded according to the Alamouti codebook $\mathcal{M}$. Finally, the sequence that has the lowest PAPR is kept and then transmitted.

Compared to the previous situation, we note that the codebook $\mathcal{M}$ is replaced by a $\left(K \times \frac{N}{2}\right)$ matrix denoted $\mathcal{G}$, where $K$ present the precoder vector sequences denoted by Cseq $^{(k)}$. Also, each component matrix , $[\mathcal{G}(k, n)]_{k=1, \ldots, K}^{n=1, \ldots, \frac{N}{2}}$, refers to 3 bits where:

1. The first bit indicates if the $X_{2 n}$ belongs to the rotated set i.e., $\mathcal{E}_{2}$ or not i.e., $\mathcal{E}_{1}$. Thus, If $\operatorname{Seq}_{n}{ }^{(k)}=0$, then $X_{2 n}^{\varphi}=X_{2 n} . e^{j \varphi_{1}}, \varphi_{1} \in E_{1}$, otherwise $X_{2 n}^{\varphi}=X_{2 n} \cdot e^{j \varphi 2}, \varphi_{2} \in E_{2}$.

2. The second bit indicates if the $X_{2 n}$ belongs $\mathcal{E}_{2}$ or $\mathcal{E}_{1}$. If $\operatorname{Seq}_{n+1}{ }^{(k)}=0$, then $X_{2 n+1}^{\varphi}=X_{2 n+1} . e^{j \varphi_{1}}, \varphi_{1} \in E_{1}$, otherwise $X_{2 n+1}^{\varphi}=X_{2 n+1} . e^{j \varphi 2}, \varphi_{2} \in E_{2}$.

3. The third bit refers to $\mathbf{S c h}_{i}, i=0$, 1. If $\operatorname{Seq}_{n+2}{ }^{(k)}=0$, then $\left(X_{2 n}^{\varphi}, X_{2 n+1}^{\varphi}\right)$ will be coded by using the scheme code $S c h_{0}$. If it is not the case, we consider $S c h_{1}$.

To simplify the analysis of the proposed technique, we suggest first to refer to the hard decision metric instead of the soft one and then we propose the use of an hierarchical coding scheme at the transmitter side assuming that $\mathcal{G}$ contains only 010 and 101 . This is equivalent to transform the hypothesis of Eq. (16) as

$\left\{\begin{array}{l}\mathcal{H}_{0}^{r}: \text { if } \mathcal{G}(i, j)=010 \Rightarrow\left(X_{2 n} \in \mathcal{E}_{1}, X_{2 n+1} \in \mathcal{E}_{2}\right) \text { and }\left(\mathbf{S c h}_{0}\right) \\ \mathcal{H}_{1}^{r}: \text { if } \mathcal{G}(i, j)=101 \Rightarrow\left(X_{2 n} \in \mathcal{E}_{2}, X_{2 n+1} \in \mathcal{E}_{1}\right) \text { and }\left(\mathbf{S c h}_{1}\right)\end{array}\right.$

Then, for $K=2$ and $N=4$, the obtained sequences is described as follows:

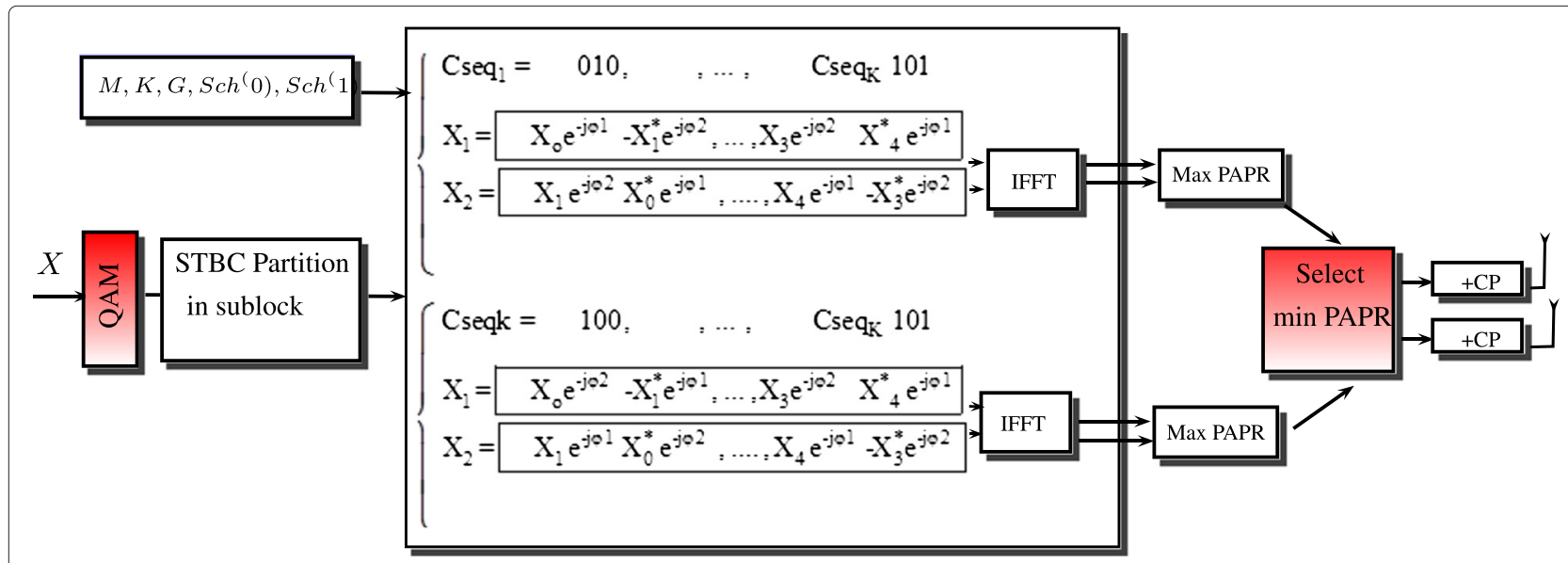

Fig. 5 Blind SLM-STBC technique: transmitter side 


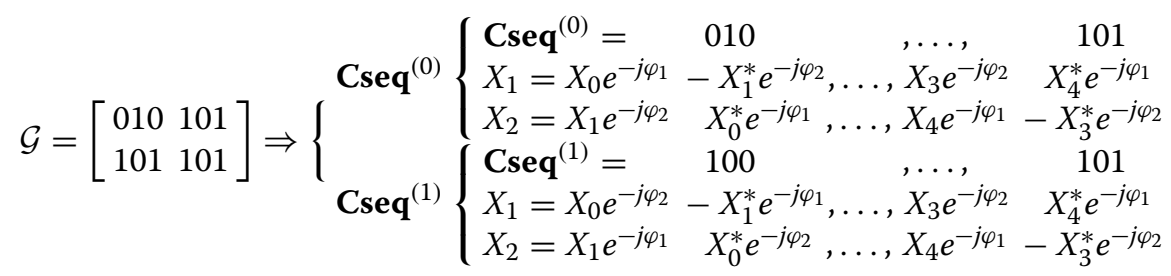

In the following section, we analyze how the actually transmitted side information index will be estimated.

\subsection{Precoder sequence $\mathrm{Cseq}^{\left(k_{0}\right)}$ estimation}

Once the precoder matrix $\mathcal{G}$ is defined, in the receiver side, we reiterate the same reasoning to recover the transmitted signal as in Section 4.1 and where we perform a hard decision. The decision process will bank on the embedded signaling defined through the STBC precoders codebook $\mathcal{G}$. Therefore, the Eq. (24) can be redefined as follows:

$$
\begin{aligned}
L L R_{n} & =\ln \left\{\frac{\operatorname{Pr}\left(\mathcal{H}_{0}^{r} \mid \mathbf{R}_{n}\right)}{\operatorname{Pr}\left(\mathcal{H}_{1}^{r} \mid \mathbf{R}_{n}\right)}\right\} \\
& =\ln \left\{\frac{\sum_{X_{2 l+1} \in \mathcal{Q}^{\prime}} \sum_{2 l \in \mathcal{Q}} \exp \left(-f\left(X_{2 l}, X_{2 l+1}\right)\right)}{\sum_{X_{2 l+1} \in \mathcal{Q}} \sum_{X_{2 l} \in Q^{\prime}} \exp \left(-g\left(X_{2 l}, X_{2 l+1}\right)\right)}\right\}
\end{aligned}
$$

where $\mathcal{Q}=\left\{q_{0}, \ldots, q_{M-1}\right\}$ and $\mathcal{Q}^{\prime}=\left\{q_{0}^{\prime}, \ldots, q_{M-1}^{\prime}\right\}$ represent the coordinates of the rotated and the unrotated constellation points, respectively.

Then, the $M L L R_{n}$ can be estimated as

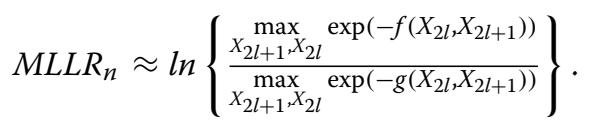

After computing the $M L L R_{n}$, the estimated vector Cseq ${ }^{\left(k_{0}\right)}$ can be constructed as

$$
\text { Cseq }{ }_{n}^{\left(k_{0}\right)}=\left\{\begin{array}{l}
010, \text { if } M L L R_{n}>=0, n=0, \ldots, \frac{N}{2}-1, \\
101, \text { else. }
\end{array} .\right.
$$

In the second step, and as the precoder matrix $\mathcal{G}$ is known at the receiver side, this vector must be mapped to the closest sequence. Therefore, the index of the considered sequence $\tilde{k}_{0}$ is deduced according to the following minimization metric:

$$
\tilde{k}_{0}=\underset{0 \leq k \leq K-1}{\arg \min }\left|\mathbf{C s e q}^{(k)}-\mathbf{C s e q}^{\left(k_{0}\right)}\right| .
$$

Finally, the transmitted sequence is deduced.

It should be noted that the complexity of our proposed methods at the receiver side is based mainly on the choice of the used STBC decoder. In our study, we consider a conventional decoder, where authors in [33] showed that the complexity of STBC detection algorithms is equal to $O\left(N^{Q}\right)$, where $N$ denotes the number of subcarriers and $Q$ denotes the number of transmitted symbols per STBC block. Thus, since we exploit in our estimation process two conventional STBC schemes, we can conclude that the complexity of our proposed methods is equal to $2 * O\left(N^{Q}\right)$.

\section{Simulation results}

The performance of the proposed blind methods in MIMO-OFDM systems is evaluated through simulation results. These simulations are performed for both the transmitter and the receiver sides. At the transmitter side, the performance is evaluated in terms of the CCDF of the PAPR versus its threshold. At the receiver side, the performances are highlighted in terms of the side information error rate (SIER) that indicates the percentage of failure detection of the phase sequence and the BER.

\subsection{Simulation parameters}

In this section, we detailed the simulation parameters based on three GPP specifications of the downlink LTE standard [36], as shown in Table1.

Besides, we consider a selective channel where the assumption of Alamouti decoder is fulfilled, which means that the channel response will be constant during the transmission period of Alamouti codeword. To achieve this assumption, we consider in the simulation, a mobile speed equal to $10 \mathrm{Km} / \mathrm{h}$, where the variations in time and in frequency of the used channel are drawn in Fig. 6.

Table 1 Parameters of simulations

\begin{tabular}{lc}
\hline Parameters & Specifications \\
\hline MIMO-OFDM system] & LTE downlink \\
Constellation & $4,16-\mathrm{QAM}$ \\
Mobile Speed $(\mathrm{Km} / \mathrm{h})$ & 10 \\
$\mathrm{Ts}(\mu \mathrm{s})$ & 72 \\
$\mathrm{fc}(\mathrm{GHz})$ & 2.15 \\
$\delta \mathrm{f}(\mathrm{KHz})$ & 15 \\
$\mathrm{~B}(\mathrm{MHz})$ & 2.5 \\
size of IFFT & 256 \\
\hline
\end{tabular}




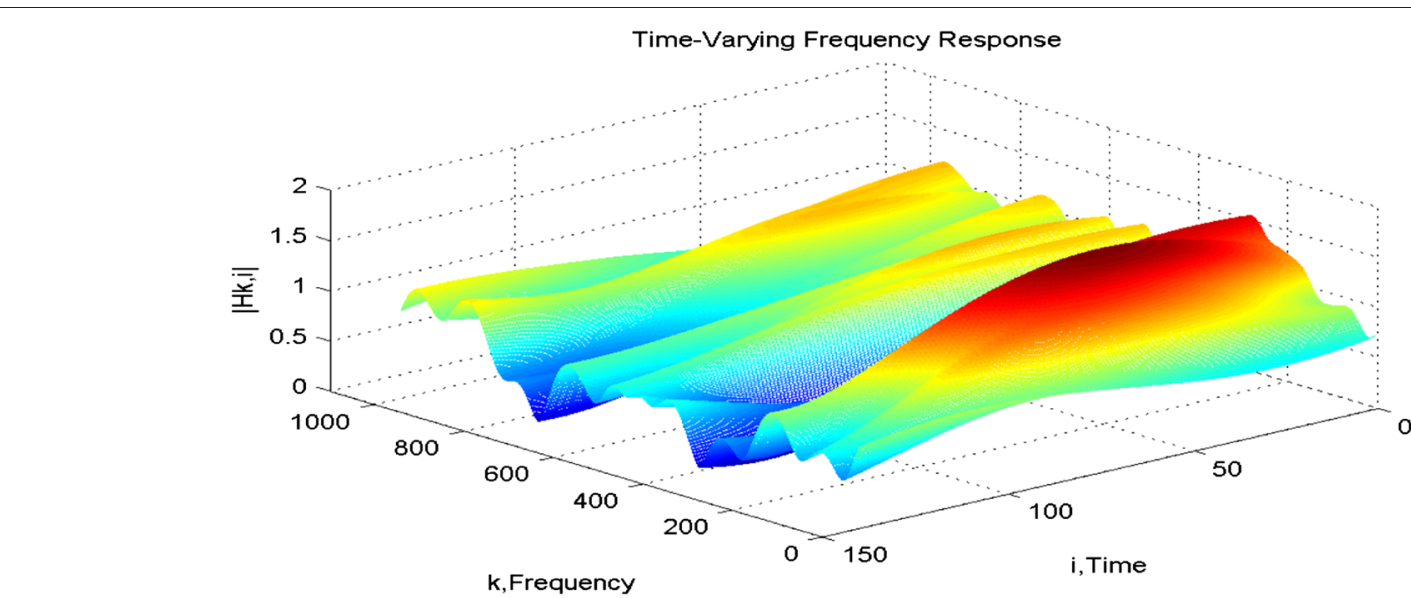

Fig. 6 Time-varying channel frequency response (mobile speed $=10 \mathrm{~km} / \mathrm{h}$ )

\subsection{Transmitter side}

In order to evaluate the performance of the proposed blind methods, we first consider the case of STBC with a full rate i.e., the rate is set to 1 , and then the case of a rate equal to $\frac{3}{4}$.
As shown in Fig. 7, we vary the number $K$ of the used precoder sequences in the STBC matrix denoted by $\mathrm{STBC}_{2}$. We deduce that when $K$ increases, the PAPR level decreases. Moreover, for $K=8$, we obtain respectively a gain of $5.5,3$, and $2.5 \mathrm{~dB}$, compared to the classical

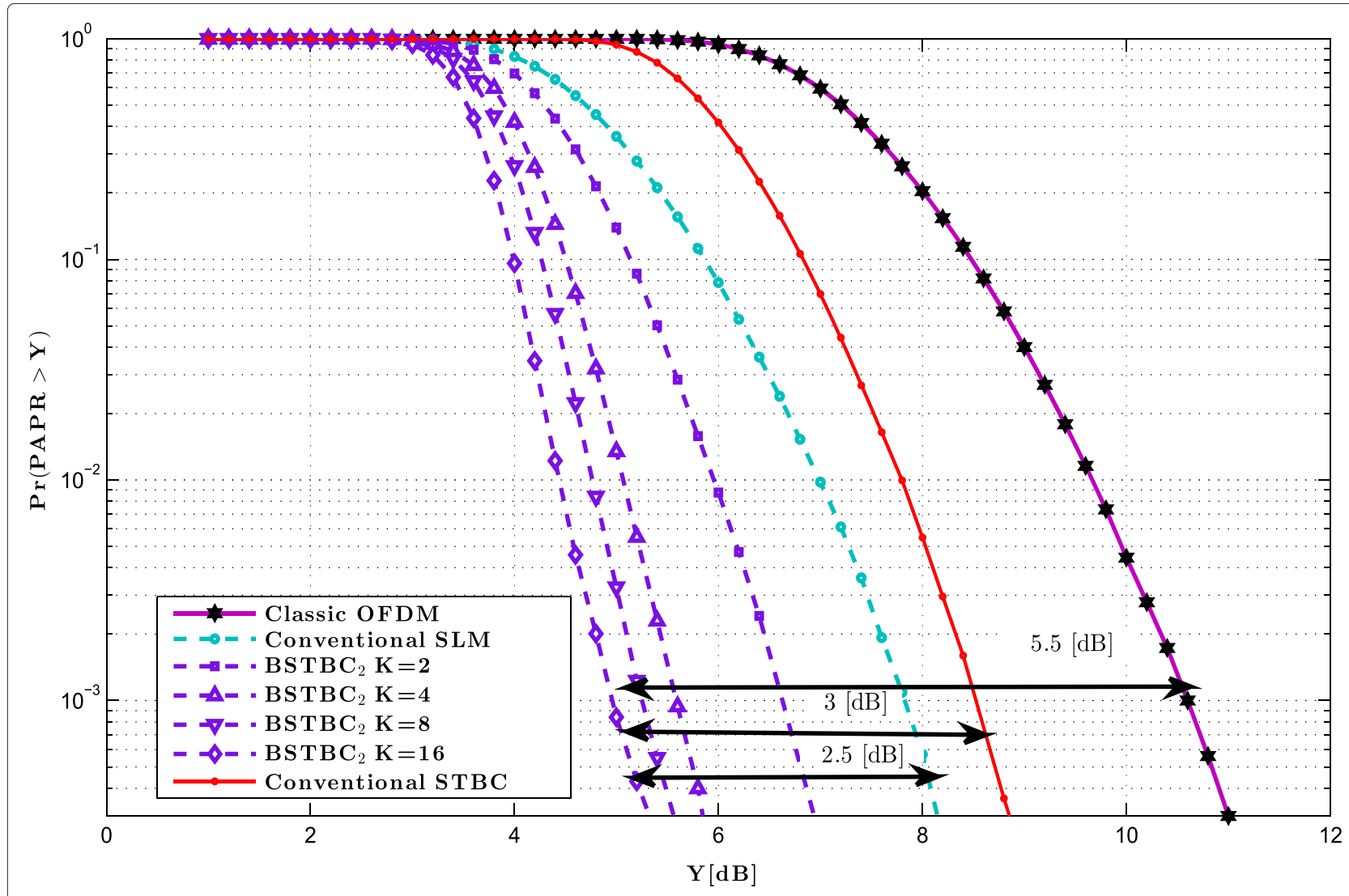

Fig. 7 Impact of the size of the STBC preorders codebook on the CCDFs of the uncoded PAPR in the case of 4-QAM and N=128 


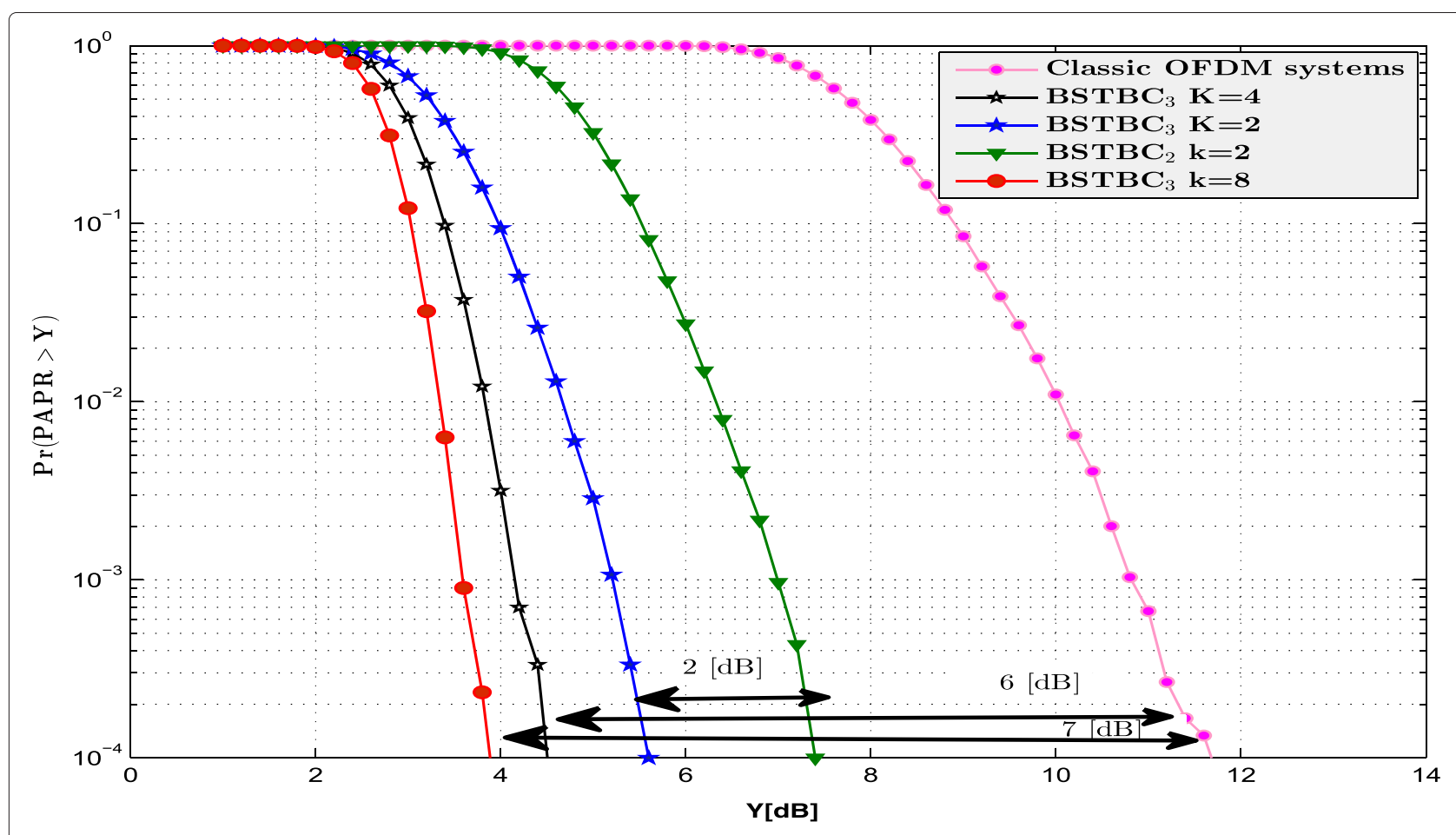

Fig. 8 Performance in terms of PAPR in the case of STBC $\frac{3}{4}$-rate for 4-QAM and $N=128$

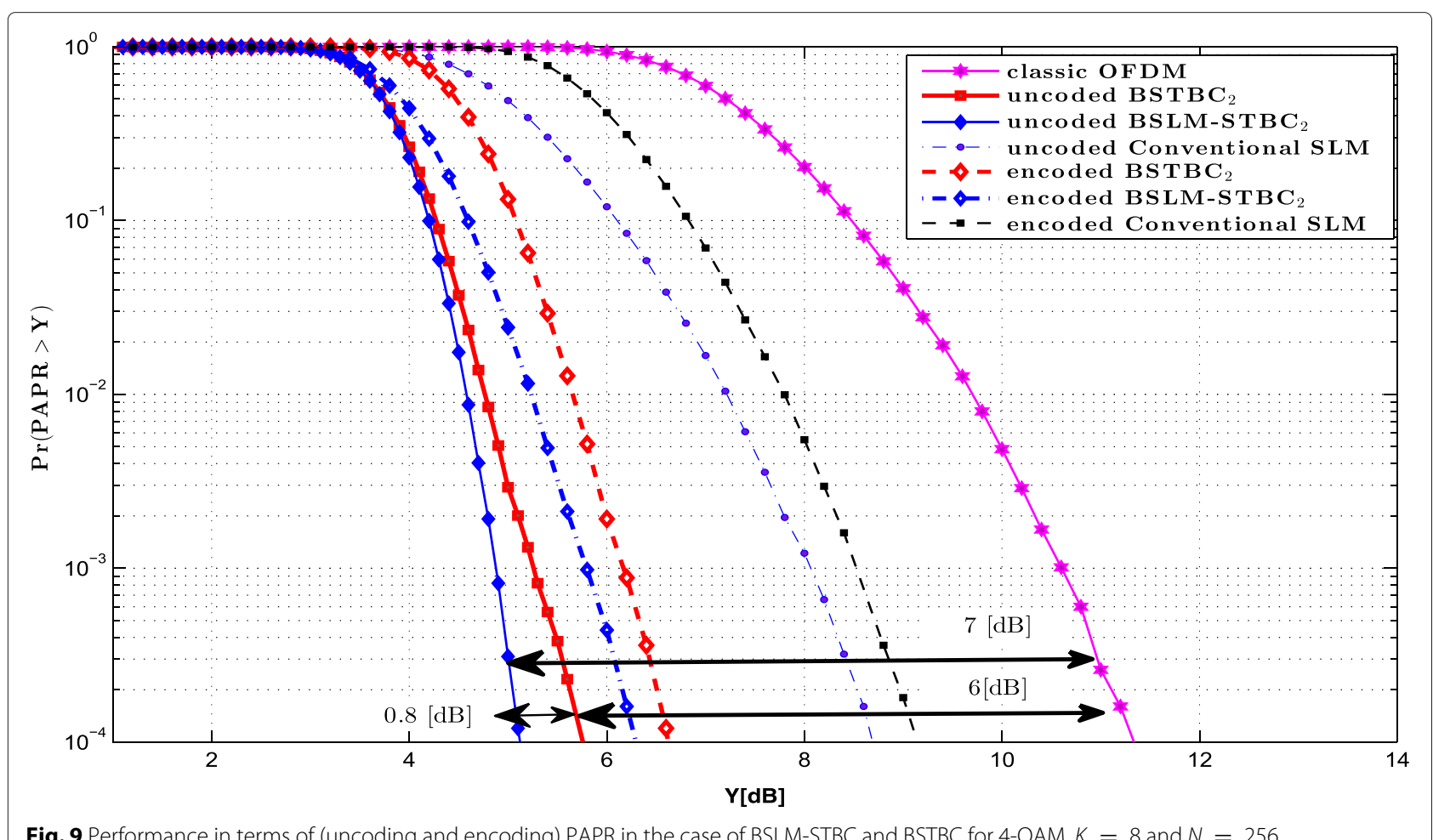

Fig. 9 Performance in terms of (uncoding and encoding) PAPR in the case of BSLM-STBC and BSTBC for 4-QAM, $K=8$ and $N=256$ 


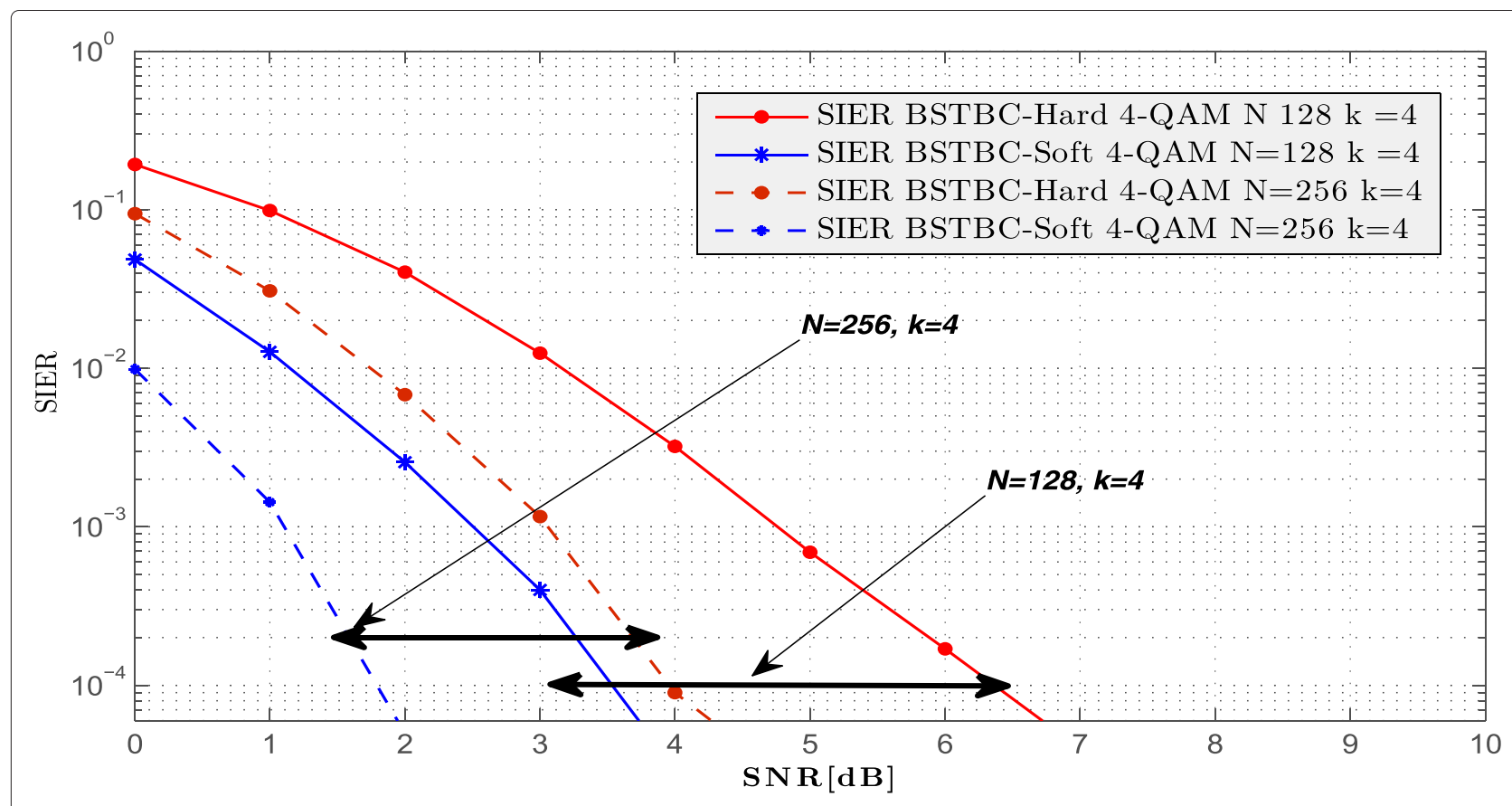

Fig. 10 Uncoded soft-BSTBC versus uncoded hard-BSTBC SIER where $K=4$ and 4-QAM

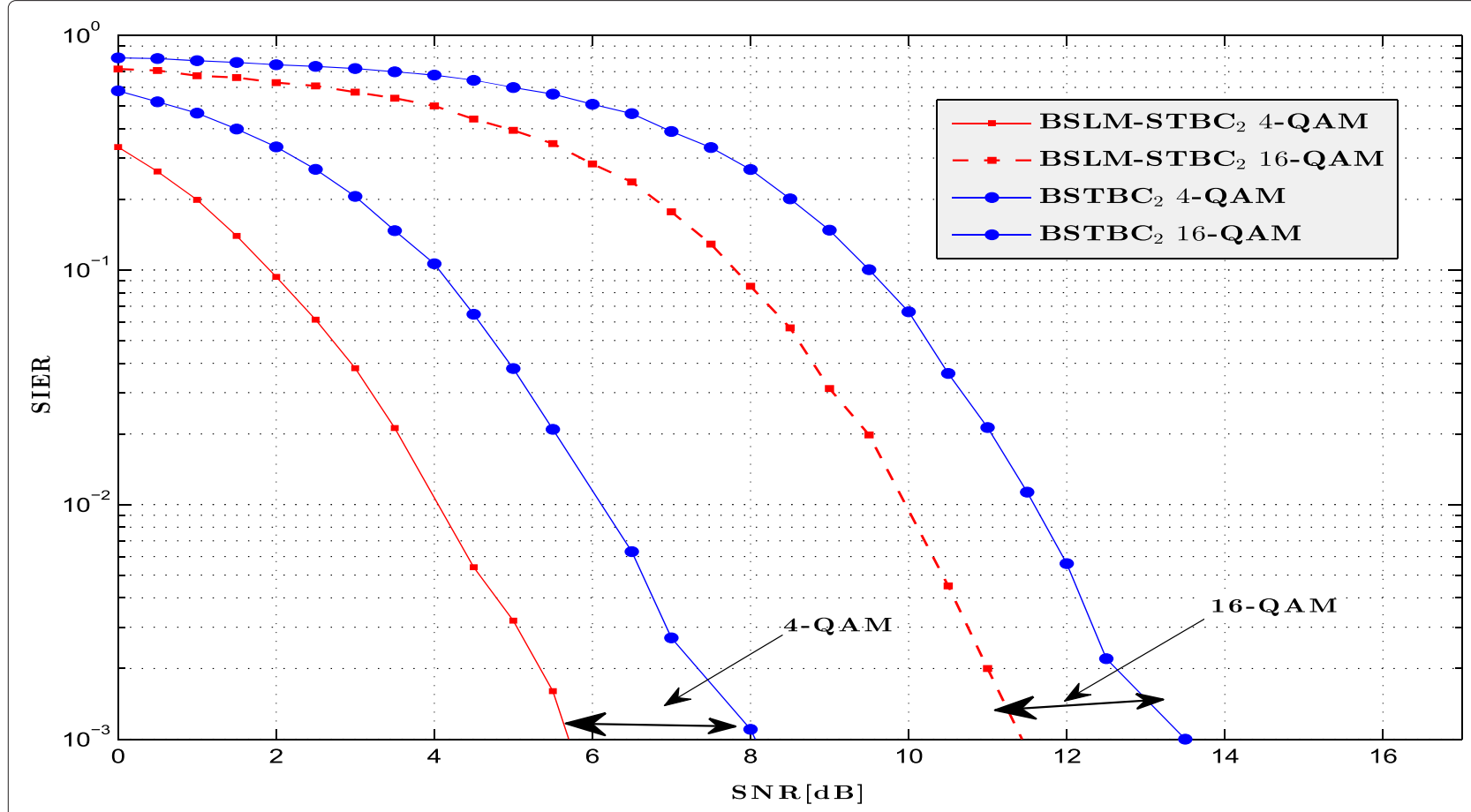

Fig. 11 Performance in terms of the uncoded SIER in the case of a Rayleigh fading channel where $K=4, N=128$, and $L=4$ for 4-QAM and 16-QAM constellations 
OFDM system, the conventional STBC and the SLM system. Using the same reasoning outlined in Section 4 and considering the schemes of the $\frac{3}{4}$-rate STBC [23, 24], the $\mathbf{S c h}_{0}$ and $\mathbf{S c h}_{1}$ can be written as

$$
\begin{aligned}
\mathbf{s c h}_{\mathbf{1}} & =\left(\begin{array}{cccc}
X_{2 n} & X_{2 n+1}^{*} & X_{2 n+2}^{*} & 0 \\
X_{2 n+1} & -X_{2 n}^{*} & 0 & X_{2 n+2}^{*} \\
X_{2 n+2} & 0 & -X_{2 n}^{*} & X_{2 n+1}^{*}
\end{array}\right), \\
\mathbf{s c h}_{\mathbf{0}} & =\left(\begin{array}{cccc}
X_{2 n} & -X_{2 n+1}^{*} & -X_{2 n+2}^{*} & 0 \\
X_{2 n+1} & X_{2 n}^{*} & 0 & -X_{2 n+2}^{*} \\
X_{2 n+2} & 0 & X_{2 n}^{*} & X_{2 n+1}^{*}
\end{array}\right) .
\end{aligned}
$$

We can observe from Fig. 8 that at a probability equal to $10^{-4}$, the Blind $\frac{3}{4}$-rate STBC method, denoted by $\mathrm{STBC}_{3}$, reduces the PAPR by 6 and $7 \mathrm{~dB}$ when $K=4$ and 8 , respectively. Furthermore, the STBC $\frac{3}{4}$-rate improves the PAPR reduction compared to the case of $\mathrm{STBC}_{2}$, where we achieve a gain around $2 \mathrm{~dB}$ for $K=2$. This gain is obtained in the case of the $\mathrm{STBC}_{3}$ since the amount of information is reduced, for each antenna, compared to the $\mathrm{STBC}_{2}$. In other words, only $\frac{3}{4}$ of data will be sent contrary to $\mathrm{STBC}_{2}$ where all the data is sent.

In Fig. 9, we consider a convolution encoder and a Viterbi decoder. In this figure, we compared the coded and the uncoded PAPR in the case of conventional SLMSTBC and the proposed Blind methods. We notice from this figure that, both methods, i.e., the encoded and the uncoded, enhance the performance in terms of PAPR compared to the conventional OFDM and the method SLM-STBC described in [17]. In addition, performing the proposed BSLM-STBC technique improves the PAPR reduction where the gain is around $0.8 \mathrm{~dB}$ for $K=8$.

\subsection{Receiver side}

Figure 10 illustrates the SIER curves for 4-QAM constellation. Clearly, we observe that both the hard and soft decision processes lead to a good system performance. Furthermore, the soft decision technique leads to a gain compared to the hard decision of $3 \mathrm{~dB}$ in the case of $N=256$.

In Fig. 11, we present the obtained SIER curves versus the SNR considering the two proposed decision techniques, ie., the one using only the hard STBC and the BSLM-STBC. We conclude that both the hardBSTBC and BSLM-STBC decisions processes lead to a good system performance. Furthermore, the BSLM-STBC decision technique leads to a significant gain compared to the hard decision technique. Indeed, in the case of Rayleigh fading channel of length $L=4$, and considering 4-QAM and 16-QAM constellations, the BSLM-STBC technique can reach to $2 \mathrm{~dB}$.

Moreover, to evaluate the performance of the receiver side in terms of BER, first, we consider two

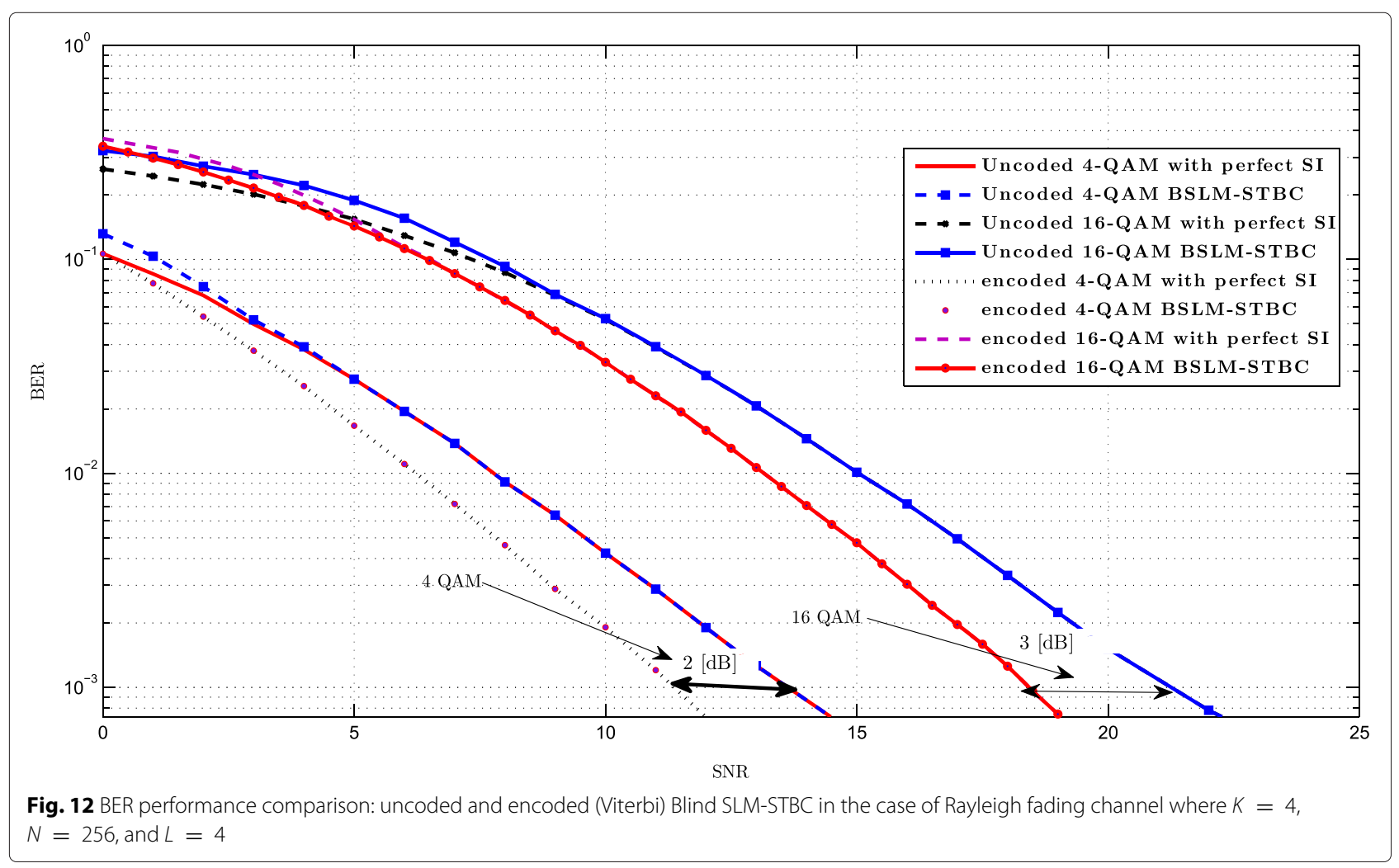




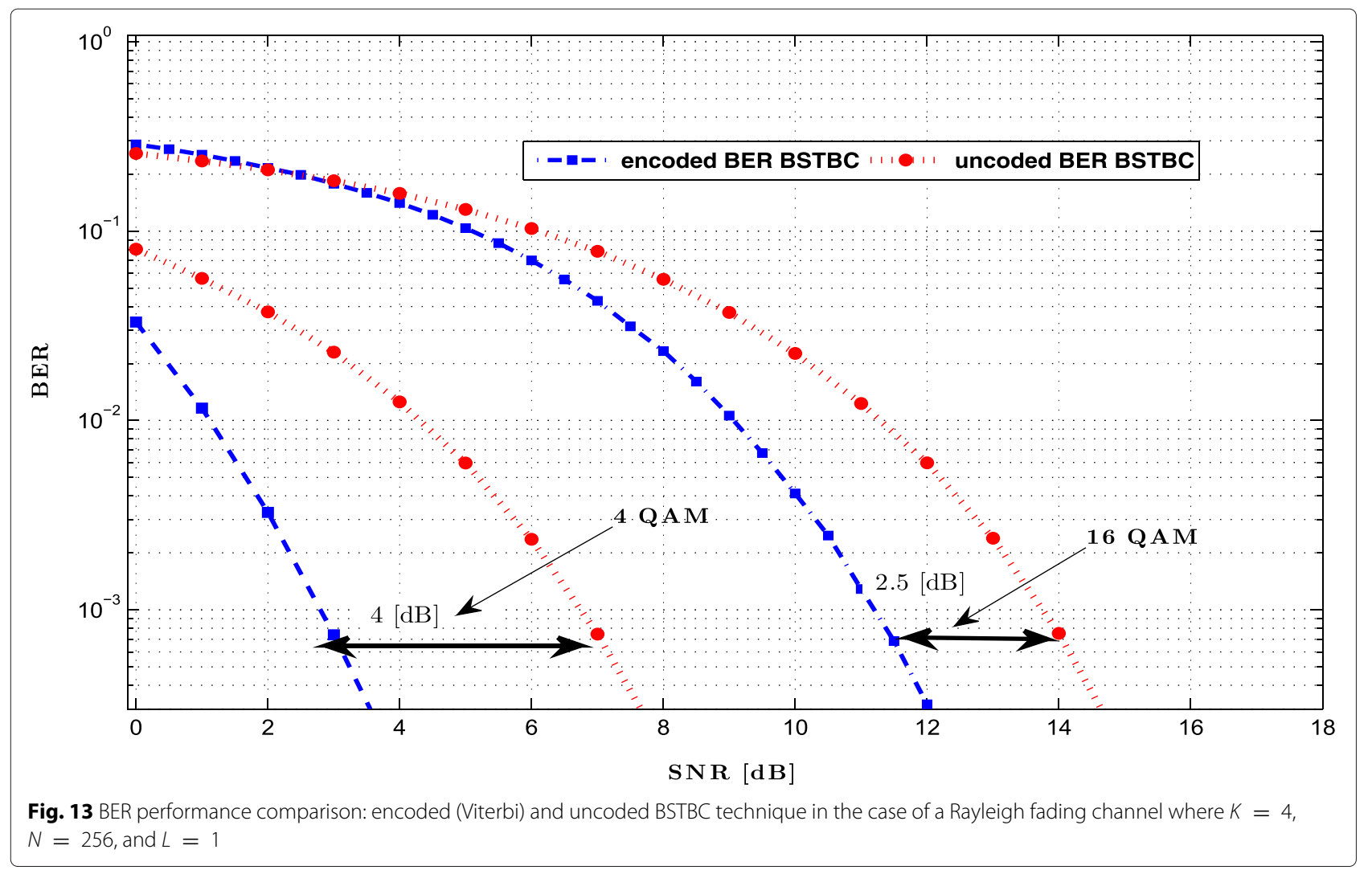

cases: the case where we perform a Blind technique (BSTBC, BSLM-STBC) and the one where the phase sequence is perfectly known at the receiver side. Second, we consider a conventional Viterbi decoder with a rate equal to $\frac{1}{2}$ and a hard decoder at the receiver side.

Figures 12 and 13 illustrate the performance of BER for convolution encoder/Viterbi decoder and uncoded Blind technique in the case of a Rayleigh fading channel where $K=4$ and $N=256$ for $L=1$ and $L=4$, respectively. For the case of QAM constellation, we observe a good fitting between the uncoded/encoded BSLM-STBC curves and the perfect uncoded/encoded classical curves. This proves well that our proposed bind methods lead to a performance which is quasi-identical to the ordinary one with perfectly known SI. Finally, we notice that using the Viterbi decoder at the receiver side enhance the performance in terms of BER, where we obtain $4 \mathrm{~dB}$ of a gain in the case of 4-QAM and $L=1$.

\section{Conclusions}

In this paper, we investigate an efficient PAPR reduction technique dedicated to the MIMO-OFDM systems using STBC precoders codebook. The main feature of our proposed method is to induce an embedded signaling through the advanced precoders codebook. This form of signaling leads to a powerful recovery of the transmitted signal and guarantees a very low failure decision rate. To further improve the decision process, we proposed an additional embedded signaling that consists of a set of rotated and unrotated QAM constellations. Indeed, when this latter is used in the decision process (using a hard decision deduced from a Max-Log-MAP decoding), it improves the MIMO-OFDM system performances in terms of the CCDF, PAPR, SIER, and BER. The work perspective will be devoted to the applicability of our proposed technique in the spectrum sensing case for the cognitive radio systems.

\section{Competing interests}

The authors declare that they have no competing interests.

\section{Acknowledgements}

Parts of this paper were published in the IEEE Wireless Communications and Networking (WCNC) 2013 conference [37].

Received: 6 March 2015 Accepted: 7 September 2015

Published online: 04 November 2015

\section{References}

1. LJ Cimini, Analysis and simulation of digital mobile channel using orthogonal frequency division multiplexing. IEEE Trans. Commun. 33(7), 665-675 (1985)

2. WY Zou, Y Wu, COFDM: an overview. IEEE Trans. Broadcast. 41(1), 1-8 (1995) 
3. YR Zheng, M Wang, W Zeng, C Xiao, Practical linear precoder design for finite alphabet multiple-input multiple-output orthogonal frequency division multiplexing with experiment validation. IET Commun. 7(9), 836-847 (2013)

4. K Zheng, B Fan, J Liu, Y Lin, W Wang, Interference coordination for OFDM-based multihop LTE-advanced networks. IEEE Wirel. Commun. 18(1), 54-63 (2011)

5. G Wunder, RFH Fischer, H Boche, S Litsyn, J-S No, The PAPR problem in OFDM transmission: new directions for a long-lasting problem. IEEE Signal Proc. Mag. 30(6), 130-140 (2013)

6. E Costa, M Midrio, S Pupolin, Impact of amplifier nonlinearities on OFDM transmission system performance. IEEE Commun. Lett. 3(2), 37-39 (1999)

7. MS Baek, MJ Kim, YH You, HK Song, Semi-blind channel estimation and PAR reduction for MIMO-OFDM system with multiple antennas. IEEE Trans, Broadcast. 50(4), 414-424 (2004)

8. MS Baek, MJ Kim, YH You, HK Song, Semi-blind channel estimation and PAR reduction for MIMO-OFDM system with multiple antennas. IEEE Trans. Broadcast. 50, 414-424 (2003)

9. M Tan, Z Latinovic, Y Bar-Ness, STBC MIMO-OFDM peak-to-average power ratio reduction by cross-antenna rotation and inversion. IEEE Commun. Lett. 9(7), 592-594 (2005). http://ieeexplore.ieee.org/xpl/login.jsp?tp= \&arnumber=1461674\&url=http\%3A\%2F\%2Fieeexplore.ieee.org\%2Fiel5 \%2F4234\%2F31451\%2F01461674

10. RFH sher, $\mathrm{M}$ Hoch, Directed selected mapping for peak-to- average power ratio reduction in MIMO OFDM. IEE Electron. Lett. 42(22), 1289-1290 (2006)

11. RFH sher, M Hoch, Partial transmit sequences for peak-to-average power ratio reduction in multiantenna OFDM. EURASIP J. Wirel. Commun. Netw. Special Issue on "Multicarrier Systems", (2010)

12. H Lee, DN Liu, W Zhu, MP Fitz, Peak power reduction using a unitary rotation in multiple transmit antennas. IEEE Int. Conf. Commun. 4, 2407-2411 (2005)

13. A Sauffr, ER Meng, Optimal peak-to-average power ratio reduction in MIMO systems. IEEE Int. Conf. Commun. (ICC). 7, 3094-3099 (2006)

14. Z Latinovic, Y Bar-Ness, SFBC MIMO-OFDM Peak-to-average power ratio reduction by polyphase interleaving and inversion. IEEE Trans. Commun. Lett. 10(4), 266-268 (2006). http://ieeexplore.ieee.org/xpl/articleDetails. jsp?arnumber $=1613742 \&$ navigation $=1$

15. CL Wang, SS Wang, HL Chang, A low-complexity SLM based PAPR reduction scheme for SFBC MIMO-OFDM systems. Proc. IEEE Wirel. Commun. Netw. Conf, 1449-1453 (2011)

16. MF Naeiny, F Marvasti, Selected mapping algorithm for PAPR reduction of space-frequency coded OFDM systems without side information. IEEE Trans. Vehi. Tech. 60(3), 1211-1216 (2011). http://ieeexplore.ieee.org/xpl/ articleDetails.jsp?arnumber $=5703137 \&$ newsearch $=$ true\&query $T e x t=$ Selected\%20Mapping\%20Algorithm\%20for\%20PAPR\%20Reduction \%20of\%20Space-Frequency\%20Coded\%200FDM\%20Systems \%20Without\%20Side\%20Information

17. MF Naeiny, PAPR Reduction scheme in SFBC MIMO-OFDM systems without side information. IEEE ICC, 4708-4712 (2013)

18. M Sghaier, F Abdelkefi, M Siala, Efficient embedded signaling through rotated modulation constellations for SLM-based OFDM systems. IEEE ICC, 3846 (2013)

19. RA Stirling Gallacher, Z Wang, Improving performance of coherent coded OFDM systems using space-time transmit diversity. Electronic Lett. 37, 457-458 (2001)

20. L Zheng, DNC Tse, Diversity and multiplexing: a fundamental trade-off in multiple-antenna channels. IEEE Trans. Inform. Theory. 49(5), 1073-1096 (2003)

21. DB KF Lee, A Williams, space-frequency transmitter diversity technique for OFDM systems. IEEE Globecom. 3, 1473-1477 (2000)

22. VTarokh, N Seshadri, AR Calderbank, Space-time codes for high data rate wireless communication: performance criterion and code construction. IEEE Trans. Inf. Theory. 44, 744-765 (1998)

23. VTarokh, H Jafarkhani, AR Calderbank, Space-time block codes from orthogonal designs. IEEE Trans. Inf. Theory. 45, 744-765 (1999)

24. VTarokh, H Jafarkhani, AR Calderbank, Space-time block coding for wireless communications: performance results. IEEE J. Sel. Areas Commun. 17, 451-460 (1999)

25. A SM Alamouti, simple transmit diversity technique for wireless communications. IEEE J. Sel. Areas Commun. 16(8), 1451-1458 (1998)
26. J Armstrong, Peak-to-average power reduction for OFDM by repeated clipping and frequency domain filtering. IET Electron. Lett. 38(5), 246-247 (2002)

27. H Ochiai, 'A novel trellis-shaping design with both peak and average power reduction for OFDM systems'. IEEE Trans. Commun. 52(11), 1916-1926, (2004) Telecommun., vol. 52, no. 1/2, pp. 58-67, Feb. 1997

28. F Abdelkefi, P Duhamel, F Alberge, Impulse noise correction in Hiperlan 2: improvement of the decoding algorithm and application to PAPR reduction. IEEE Int. Conf. Commun. (ICC). 3, p2094-2098 (2003)

29. T Jiang, Y Zhou, An improved tone reservation scheme with fast convergence for PAPR reduction in OFDM systems. IEEE Trans. Broadcast. 57(4), 902-906 (2011)

30. DW Lim, SJ Heo, JS No, On the phase sequence set of SLM OFDM scheme for a crest factor reduction. IEEE Trans. Signal Proc. 12(2), 93-96 (2005)

31. L Yang, KK Soo, SQ Li, YM Siu, P A P R reduction using low complexity PTS to construct of OFDM signals without side information. IEEE Trans. Broadcast. 57(2), 284-290 (2010)

32. L Hanzo, M Munster, BJ Choi, T Keller, OFDM and MC-CDMA for broadband multi-user communications, WLANs and Broadcasting. (IEEE Press and John Wiley \& Sons, Ltd, Chichester UK, 2003)

33. C Xu, D Liang, Reduced-complexity soft STBC detection. IEEE Globecom. 57(2), 42170-4221 (2012)

34. K Fazel, S Kaiser, Multi-carrier and spread spectrum systems, 2nd ed. (John Wiley, Sons, United Kingdom, 2008)

35. Y Lu, G Stuber, Orthogonal Frequency Division Multiplexing for Wireless Communications. (Springer, Atlanta, USA, 2006)

36. 3rd Generation Partnership Project, Technical Specification Group Radio Access Network; Evolved Universal Terrestrial Radio Access (UTRA): Base Station (BS) Radio Transmission and Reception, TS 36.104. V8.7.0 September 2009

37. M Sghaier, F Abdelkefi, M Siala, Efficient embedded signaling through Alamouti STBC precoders in MIMO-OFDM systems, (WCNC), 4053-4058 (2013). http://ieeexplore.ieee.org/xpl/login.jsp?tp=\&arnumber= 6555226\&url=http\%3A\%2F\%2Fieeexplore.ieee.org\%2Fxpls\%2Fabs_all.jsp \%3Farnumber\%3D6555226

\section{Submit your manuscript to a SpringerOpen ${ }^{\circ}$ journal and benefit from:}

- Convenient online submission

- Rigorous peer review

- Immediate publication on acceptance

- Open access: articles freely available online

- High visibility within the field

- Retaining the copyright to your article

Submit your next manuscript at $\gg$ springeropen.com 